ARTICLE OPEN

\title{
NF1 deficiency correlates with estrogen receptor signaling and diminished survival in breast cancer
}

Patrick S. Dischinger ${ }^{1}$, Elizabeth A. Tovar ${ }^{1}$, Curt J. Essenburg ${ }^{1}$, Zachary B. Madaj ${ }^{2}$, Eve E. Gardner ${ }^{1}$, Megan E. Callaghan ${ }^{1}$, Ashley N. Turner iD $^{3}$, Anil K. Challa ${ }^{3}$, Tristan Kempston ${ }^{4}$, Bryn Eagleson ${ }^{4}$, Robert A. Kesterson ${ }^{3}$, Roderick T. Bronson $^{5}$, Megan J. Bowman $^{2}$, Carrie R. Graveel ${ }^{1}$ and Matthew R. Steensma ${ }^{1,6,7}$

The key negative regulatory gene of the RAS pathway, NF1, is mutated or deleted in numerous cancer types and is associated with increased cancer risk and drug resistance. Even though women with neurofibromatosis (germline NF1 mutations) have a substantially increased breast cancer risk at a young age and NF1 is commonly mutated in sporadic breast cancers, we have a limited understanding of the role of NF1 in breast cancer. We utilized CRISPR-Cas9 gene editing to create Nf1 rat models to evaluate the effect of $N f 1$ deficiency on tumorigenesis. The resulting Nf1 indels induced highly penetrant, aggressive mammary adenocarcinomas that express estrogen receptor (ER) and progesterone receptor (PR). We identified distinct Nf1 mRNA and protein isoforms that were altered during tumorigenesis. To evaluate NF1 in human breast cancer, we analyzed genomic changes in a data set of 2000 clinically annotated breast cancers. We found NF1 shallow deletions in $25 \%$ of sporadic breast cancers, which correlated with poor clinical outcome. To identify biological networks impacted by NF1 deficiency, we constructed gene co-expression networks using weighted gene correlation network analysis (WGCNA) and identified a network connected to ESR1 (estrogen receptor). Moreover, NF1-deficient cancers correlated with established RAS activation signatures. Estrogen-dependence was verified by estrogen-ablation in Nf1 rats where rapid tumor regression was observed. Additionally, Nf1 deficiency correlated with increased estrogen receptor phosphorylation in mammary adenocarcinomas. These results demonstrate a significant role for NF1 in both NF1related breast cancer and sporadic breast cancer, and highlight a potential functional link between neurofibromin and the estrogen receptor.

npj Breast Cancer (2018)4:29; doi:10.1038/s41523-018-0080-8

\section{INTRODUCTION}

Deregulated RAS signaling promotes several "hallmarks of cancer", such as sustained proliferation, invasion, metastasis, and angiogenesis. ${ }^{1}$ The importance of RAS deregulation in cancer is demonstrated by the fact that KRAS is the most commonly mutated oncogene and occurs at a high frequency in lung (17\%), pancreatic (57\%), and colon (33\%) cancers. ${ }^{2}$ Even though KRAS is mutated in only $4 \%$ of sporadic breast cancers (HRAS $=1 \%$; NRAS $=2 \%)^{2}$ the RAS/ERK pathway is hyperactivated in approximately $50 \%$ of breast cancers. ${ }^{3-5}$ This discrepancy suggests that there is another mechanism underlying RAS activation, besides mutation, in human breast cancers.

The key negative regulatory gene of the RAS pathway, NF1, is mutated or deleted in a wide range of cancers and is increasingly recognized as a significant cancer driver. The NF1 gene encodes neurofibromin, a GTPase-activating protein that regulates RAS (including HRAS, NRAS, and KRAS). Loss of neurofibromin function results in hyperactivated RAS. ${ }^{6}$ Neurofibromin is a large 2818 amino acid protein in which exons 20-27 encode for the GAPrelated domain (GRD). ${ }^{7}$ Neurofibromin functions as a monomer and stimulates intrinsic RAS GTPase activity through Spred1- mediated binding to the GRD in K-RAS, N-RAS, and H-RAS. Mutations or deletions within the GRD result in decreased neurofibromin functionality and deregulated RAS signaling driven through the RAS-RAF-MEK-ERK cascade., ${ }^{6,8}$ Neurofibromin contains other functional domains; however, the role of these domains and others in tumor suppression is undefined. ${ }^{8}$

Neurofibromatosis type 1 (NF) is caused by germline mutations in the NF1 gene and is the most common single-gene disorder affecting 1 in 3000 live births. ${ }^{9,10}$ The majority of NF patients develop benign cutaneous neurofibromas and may also develop peripheral nerve tumors, neurocognitive disorders, and bone stigmata (tibial dysplasia, scoliosis, and osteoporosis). Importantly, NF patients have an increased risk of developing several adult cancers including breast, ovarian, liver, lung, bone, thyroid, and gastrointestinal. ${ }^{11}$ These diverse clinical manifestations reveal the impact of loss of NF1 function and dysregulated RAS in numerous tissue types.

In addition to germline mutations, NF1 mutations and deletions commonly occur in sporadic cancers and are associated with increased cancer risk and drug resistance. NF1 is the third most prevalent mutated or deleted gene in glioblastoma, ${ }^{12}$ fourth most

\footnotetext{
${ }^{1}$ Center for Cancer and Cell Biology, Van Andel Research Institute, Grand Rapids, MI, USA; ${ }^{2}$ Bioinformatics \& Biostatistics Core, Van Andel Research Institute, Grand Rapids, MI, USA; ${ }^{3}$ Department of Genetics, The University of Alabama at Birmingham, Birmingham, AL, USA; ${ }^{4}$ Vivarium and Transgenics Core, Van Andel Research Institute, Grand Rapids, MI, USA; ${ }^{5}$ Rodent Histopathology Core Dana Farber/Harvard Cancer Center, Harvard Medical School, Boston, MA, USA; ${ }^{6}$ Helen DeVos Children's Hospital, Spectrum Health System, Grand Rapids, MI, USA and ${ }^{7}$ Michigan State University College of Human Medicine, Grand Rapids, MI, USA

Correspondence: Carrie R. Graveel (carrie.graveel@vai.org) or Matthew R. Steensma (matt.steensma@vai.org)

These authors contributed eqully: Carrie R. Graveel, Matthew R. Steensma
}

Received: 1 March 2018 Revised: 11 July 2018 Accepted: 23 July 2018

Published online: 30 August 2018 
mutated gene in ovarian cancer, ${ }^{13}$ and the second most common mutated tumor suppressor in lung adenocarcinoma. ${ }^{14}$ More recently, clinical evidence has mounted demonstrating that women with NF have a significantly increased breast cancer risk. A risk analysis of $3672 \mathrm{NF}$ patients found that women with NF have an increased relative risk of developing breast cancer in their younger years compared to the general population (relative risk was 6.5 at 30-39 years, 4.4 at $40-49$ years, and 2.6 at $50-59$ years). ${ }^{15}$ Another key study of 1,404 NF patients identified an unequivocal increased risk for breast cancer with standardized incidence ratios of $11.1(95 \% \mathrm{Cl}, 5.56-19.5)$ for breast cancer in women with NF age $<40$ years and the overall breast cancer mortality ratio was $5.20(95 \% \mathrm{Cl}, 2.38-9.88) .{ }^{16}$ This study, in addition to several others, has established the increased breast cancer risk and associated poor outcome in patients with neurofibromatosis. ${ }^{17}$ Comprehensive genomic analyses of sporadic breast cancers revealed that NF1 is commonly mutated and may be an important driver in sporadic breast cancer. ${ }^{18,19}$ A study using the $\mathrm{Mcm} 4^{\text {Chaos } 3}$ mouse model of chromosomal instability identified $\mathrm{NF1}$ deletions in almost all of the $M c m 4^{\text {Chaos } 3}$ mammary adenocarcinomas. $^{20}$ These findings suggest that NF1 is a critical tumor suppressor and potential driver of breast tumorigenesis.

Much of our understanding of the mechanisms underlying the functional loss of NF1 and tumorigenesis come from studies of genetically engineered mouse models. ${ }^{21-24}$ These models have been valuable in defining how NF1 loss and deregulated RAS/ MAPK signaling promote tumorigenesis. However, mouse models have general limitations, particularly with respect to reproducing human pharmacokinetics and recreating putative interactions between genetic/environmental factors and induced gene deficiency. ${ }^{25}$ It is well established that mice with germline heterozygous Nf1 mutations do not spontaneously express key aspects of the human phenotype and require additional crosses into other germline mutants such as Tp53, or cell-specific conditional Nf1 mutation, to elicit more representative phenotypes. ${ }^{22,26-30}$ Prior to the demonstration of CRISPR-Cas9 gene editing, manipulation of the rat genome was far more difficult than the mouse. The rat offers significant advantages over the mouse because of its larger size, more representative physiology to human disease, higher degree of cognition and memory, and ease of use in pharmaceutical studies. ${ }^{31,32}$ In our study we utilized CRISPR-Cas9 gene editing capabilities to create multiple congenic models of Nf1 deletions in order to evaluate the effect of $N f 1$ deficiency on tumorigenesis. The resulting $N f 1$ indels affected transcription by generating both in-frame and out-of-frame sequence variation. Both mutation types were associated with highly penetrant, aggressive mammary adenocarcinomas in multiple rat founder lines. Phenotype penetrance and expression extended through second generation breeding including mammary adenocarcinomas in both Nf1-deficient males and females.

To evaluate the impact of NF1 in sporadic breast cancer we analyzed genomic changes in a large breast cancer data set composed of $>2000$ clinically annotated breast cancers. We found that NF1 shallow deletions are present in $25 \%$ of sporadic breast cancers and correlated with poor clinical outcome. ${ }^{33}$ To identify biological networks impacted by NF1 deficiency, we constructed gene co-expression networks using weighted gene correlation network analysis (WGCNA) and identified co-expression networks. A module associated with NF1 shallow deletion contained several genes that are considerably important in both ER + breast cancers and endocrine resistance, including ESR1 and FOXA1. Unsupervised hierarchical clustering revealed that breast cancers with NF1 shallow deletions form a distinct cluster that correlate with estrogen receptor (ER)-negative breast cancer and RAS activation. We also validated estrogen dependence of Nf1-deficient breast cancers by ablating mammary tumors in our Nf1 rat model by ovariectomy. These results demonstrated the significant role NF1 plays in both NF1-related breast cancer and sporadic breast cancer. Moreover, this novel Nf1 rat model is invaluable for interrogating the role of NF1, estrogen-dependent breast cancer, and deregulated RAS signaling in sporadic and inherited breast cancer.

\section{RESULTS}

Establishing Nf1 rat models using CRISPR-Cas9 endonucleases

To investigate the effects of altered $N f 1$ function in rat, we used two unique sgRNAs to target the GRD region in exon 20 and disrupt neurofibromin function (Fig. 1a). Two unique CRISPR/ sgRNAs were synthesized and co-injected with Cas9 mRNA into one-cell-stage Sprague-Dawley rat embryos, which were then transferred to pseudopregnant females. From two rounds of injections, 19 pups were born. Genotyping was performed by amplifying a $452 \mathrm{bp}$ fragment encompassing the sgRNA target sites from DNA isolated from tail biopsies, followed by a heteroduplex mobility assay (HMA). ${ }^{34}$ PCR amplicons with distinct HMA profiles were cloned into a plasmid vector and plasmids from multiple individual colonies were sequenced to identify the genetic lesions resulting from non-homologous end joining (NHEJ). Based on HMA profiles, indels were identified in 18 of 19 pups (Supplementary Tables 1 and 2). Multiple alleles were present in the majority of the G0 animals and were confirmed by Sanger sequencing, which revealed 34 mutant alleles, including 25 unique mutant alleles (Supplementary Table 2). Of the 34 mutations observed, $25(73.5 \%)$ were frameshift mutations in both the $5^{\prime}$ and $3^{\prime}$ CRISPR target sites; 14 mutations (41.2\%) were deletions ranging from 54-63 bp, spanning both the target sites. Interestingly, all the large deletions were in-frame with the translated protein coding sequence (Fig. 1b, c). HMA profiles of multiple $\mathrm{G} 0$ animals revealed the presence of additional, smaller indels at the $5^{\prime}$ and $3^{\prime}$ CRISPR target regions (Fig. 1 b, i.e. lanes 3, 8, 10 , etc) as have been observed in other studies. ${ }^{34,35}$ To verify the presence of the smaller indels and the larger in-frame deletions in each animal, we employed a three-step process of HMA, restriction digest, and Sanger sequencing (Fig. 1d, e, Supplementary Table 1, 2). The majority of smaller indels 10/19 (52.6\%) were detected in the $5^{\prime}$ CRISPR target region of G0 animals. Within the $5^{\prime}$ CRISPR target region, there is a unique Hpy81 restriction site that is lost in each of the smaller indels. Consequently, PCR amplification and Hpy81 restriction digestion was used to confirm the presence of the smaller indels in each animal (Fig. 1d). Each of these small indels at the $5^{\prime}$ CRISPR target region resulted in premature stop codons in all cases (Fig. 1e, Supplementary Table 1). Indels were also observed in the $3^{\prime}$ CRISPR target region in 5/19 (26.3\%) animals; however, these were not predicted to have an effect on protein translation due to the presence of premature stop codons at the $5^{\prime}$ CRISPR target region.

To investigate the effects of both the $N f 1$ in-frame (referred to as IF) and premature stop (referred to as PS) indels, we bred four founder rats ( 2 male and 2 female) that carried a mix of indels consisting of in-frame deletions and premature stops with wildtype Sprague-Dawley rats (Fig. 1 and Supplementary Table 1). From two male founders, we generated two unique lines: $N f 1^{\mid F-57 /+}$ from male founder \#2 and $N f 1^{I F-57 / P S-8}$ derived from male \#3. From two female founders, the lines $N f 1^{I F-63 / P S-11}$ (founder \#10) and Nf1 ${ }^{I F-}$ 54/PS-11 (founder \#13) were generated. Segregation of the IF and PS alleles occurred in the G1 generations for lines $N f 1^{\mid F-57 / P S-8}$ and $N f 1^{I F-54 / P S-11}$, resulting in $N f 1^{P S-8 /+}, N f 1^{P S-11 /+}, N f 1^{1 F-57 /+}$ lines (male $\# 3)$. These $N f 1$ lines were bred out for the following studies.

Upon performing PCR-HMA and sequence analysis, we observed the presence of more than two Nf1 alleles in individual animals. In Nf1 $1^{I F-63 / P S-11}$ we identified 3 Nf1 alleles: a WT allele, an allele with an $11 \mathrm{bp}$ deletion (premature stop), and an allele with a $-63 \mathrm{bp}$ deletion (Supplementary Fig. 1, Supplementary Table 1). The presence of more than 2 alleles was confirmed independently 


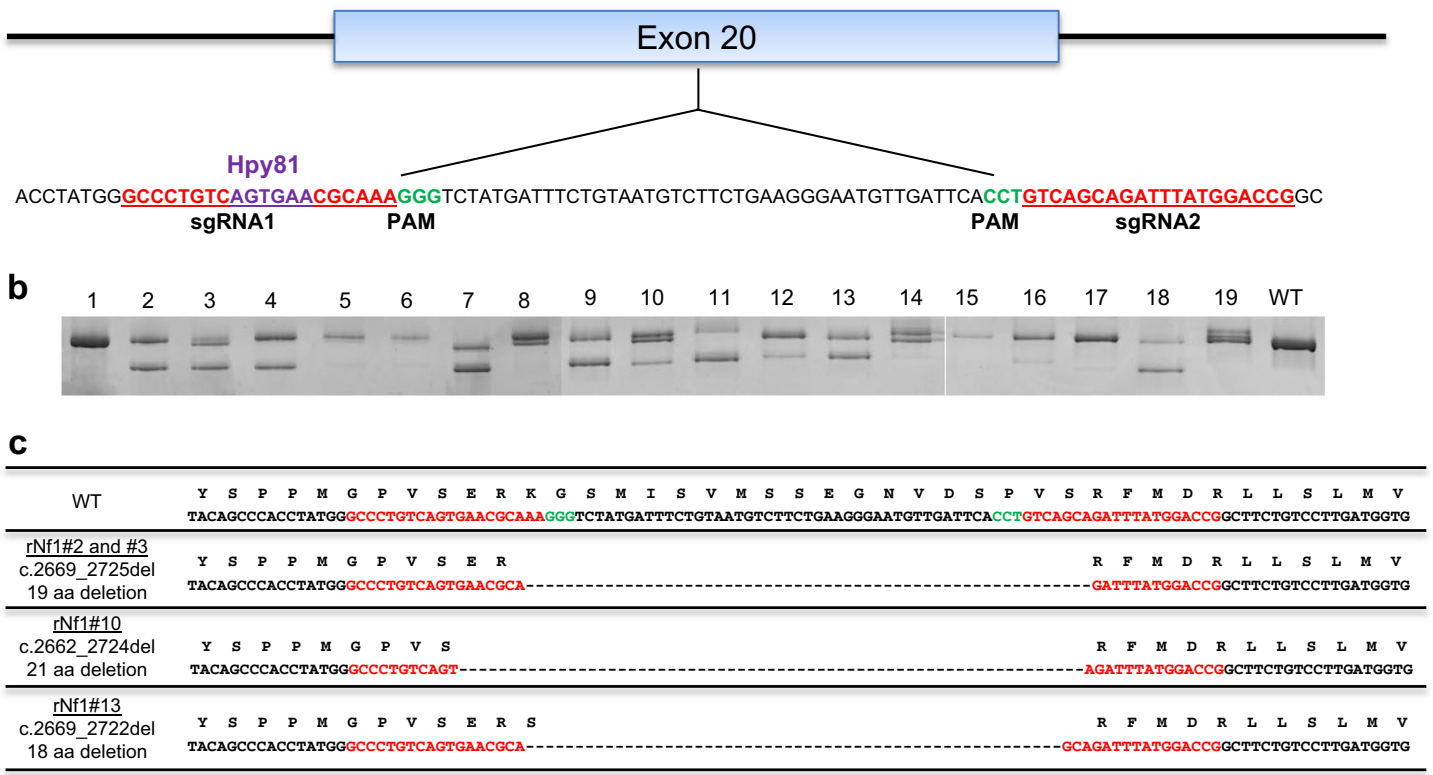

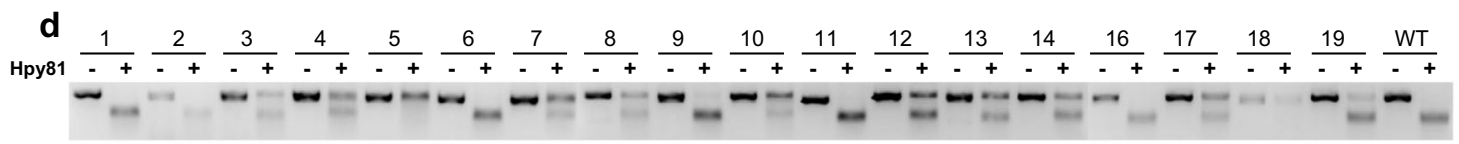

e

\begin{tabular}{|c|}
\hline 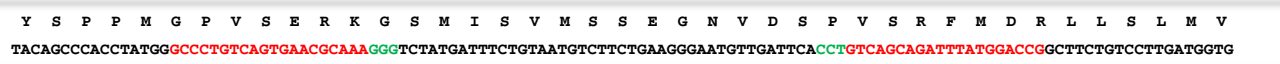 \\
\hline 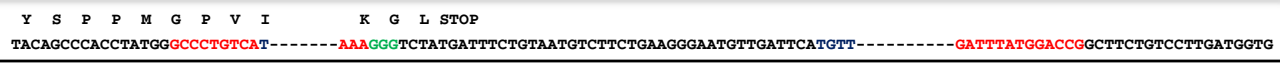 \\
\hline 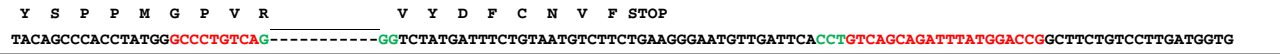 \\
\hline
\end{tabular}

Fig. 1 In-frame and premature indels are present in Nf1 G0 rats. a CRISPR-Cas9 targeting of the Nf1 GRD domain in exon 20 using two sgRNAs (red). PAM sequences (green) and the Hpy81 site (purple) used for HMA/restriction digest analysis are shown. b Heteroduplex PCR amplicons from $19 \mathrm{Nf1} \mathrm{GO}$ animals. c DNA and peptide sequence alignment of the in-frame deletions within Nf1 exon 20. d Heteroduplex PCR amplicons from $19 \mathrm{Nf1}$ G0 animals were digested with Hpy81 to reveal the presence of smaller indels. No change between the $-\mathrm{Hpy} 81$ and $+\mathrm{Hpy} 81$ indicates the Hpy81 restriction digestion was lost during CRISPR recombination events. e DNA and peptide sequence alignment of the small indels at the $5^{\prime}$ and $3^{\prime}$ CRISPR target region

by HMA and sequencing analysis using unique primer sets in two separate labs. Furthermore, these 3 alleles were transmitted through the germline in $G 1$ and G2 generations and did not segregate in either generation (Supplementary Fig. 1). Even though this finding was unanticipated, this is not unique in that the rat genome is known to have additional allelic copies of other genes. $^{36,37}$

Nf1 female and male rats develop aggressive mammary adenocarcinomas

$N f 1$ rats (including in-frame and premature stop indels) were aged to determine the effects of $\mathrm{NF} 1$ alterations on tumor development. In $\mathrm{Nf} 1$ female rats, we observed aggressive, multifocal tumorigenesis in the mammary glands at a young age (6-8 weeks). Mammary tumors were observed in $100 \%(11 / 11)$ of G0 females at the average age of 51 days (Supplementary Table 1). Tumor development progressed rapidly in all but one female (rNf1\#19), whereas the majority of G0 females had to be euthanized before reaching a mature breeding age. We were able to breed two females before their tumor burden required euthanasia ( $r N f 1 \# 10$ and \#13) and support the pups through fostering. Multiple mammary adenocarcinomas (2-8) were observed in each animal and were detected in mammary glands 1-6. The detailed tumor pathology for each animal is described in Supplementary Table 3. Notably, two male founders ( $r N f$ \#2 and \#3) developed mammary tumors at 14-16 months of age. Male \#2 developed four unique mammary adenocarcinomas, whereas male \#3 developed one mammary adenocarcinoma but had to be killed due to a larger fibrous histiocytic sarcoma. This aggressive mammary phenotype was highly penetrant and multifocal mammary tumors were observed in the subsequent G1 and G2 generations from each of the $N f 1$ lines, including lines derived from male founders (Supplementary Table 3).

Histopathologic analysis of the mammary tumors revealed that both Nf1 in-frame deletions and premature stop indels induced a wide variety of histopathologic mammary tumor types. Mammary tumors with acinar, solid, ductular, and cystic histology were observed in tumors in Nf1 female rats from each line (Fig. 2a). Moreover, Nf1 animals often developed mammary adenocarcinomas with mixed histology such as acinar and solid (Fig. 2a, left panel); cystic, papillary, ductal, and solid (Fig. 2a, middle panel); 

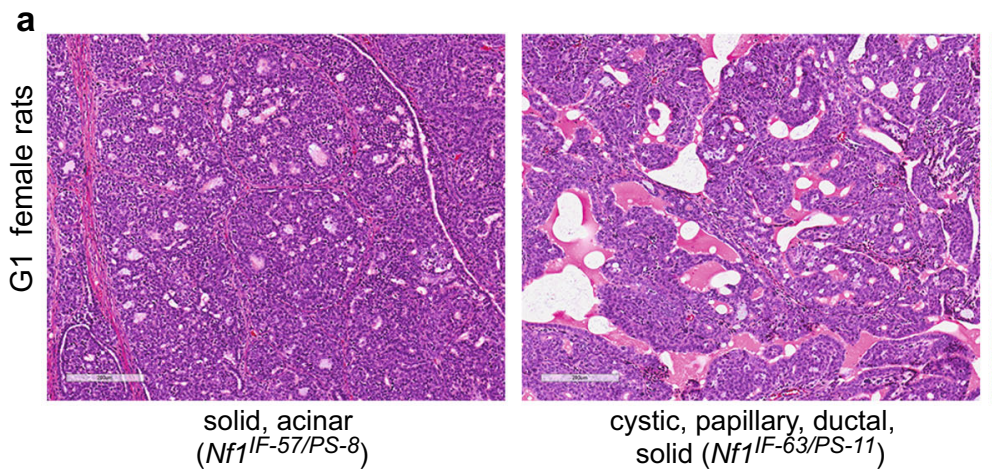

cystic, papillary, ductal, solid $\left(N f 1^{1 F-63 / P S-11}\right)$

b

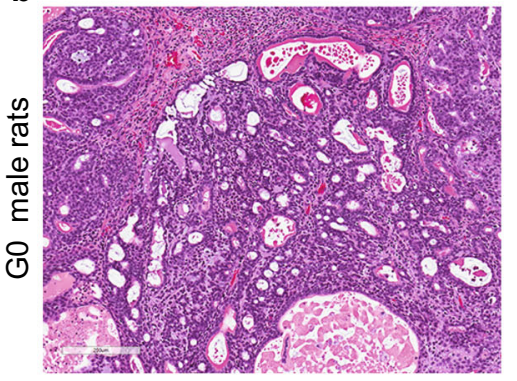

acinar and ductal $\left(N f 1^{I F-57 /+}\right)$

C

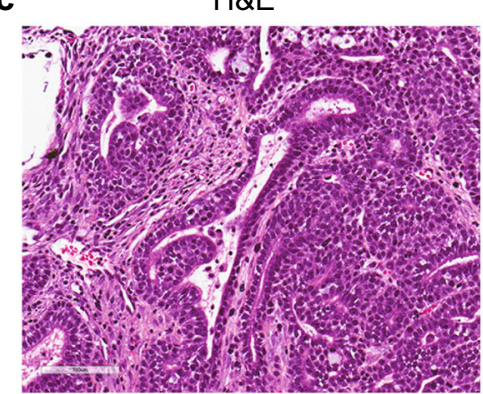

HER2/Neu

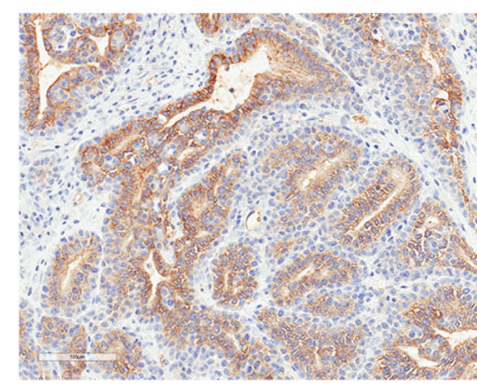

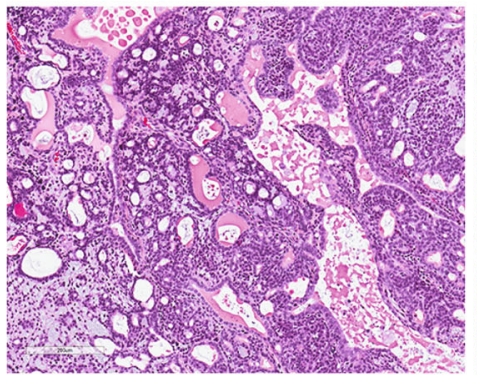

acinar and secretory acinar $\left(\mathrm{Nf} 1^{\mid \mathrm{F}-57 /+}\right)$

ER

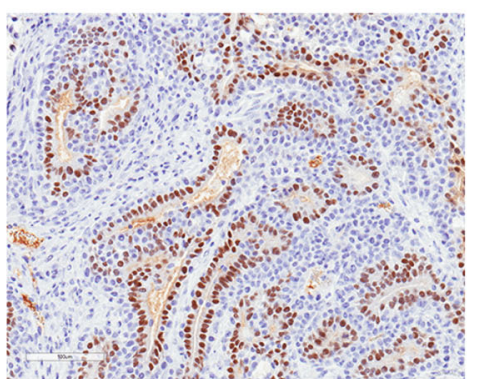

$\mathrm{Ki} 67$

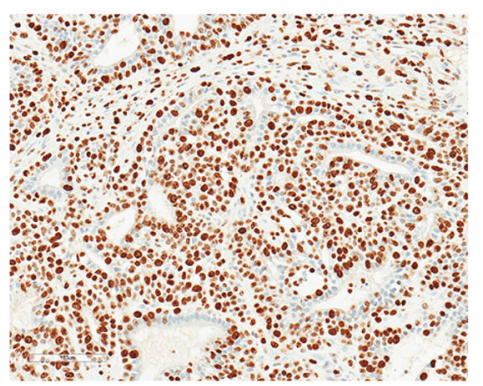

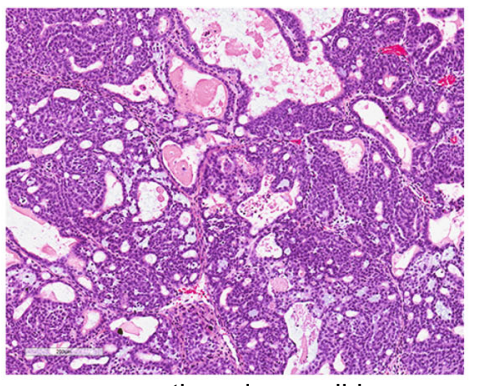

cystic, acinar, solid

$\left(N f 1^{I F-54 / P S-11}\right)$

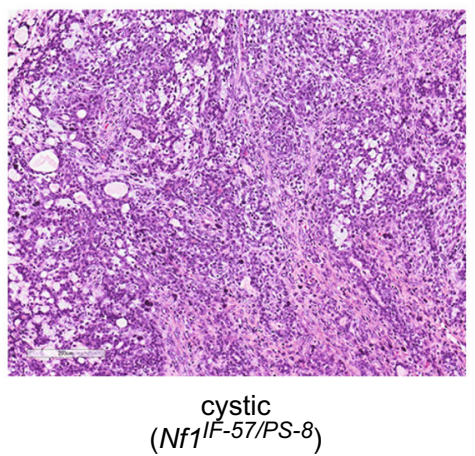

PR

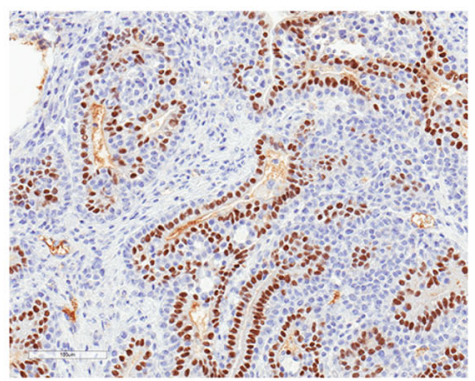

CK (AE1/AE3)

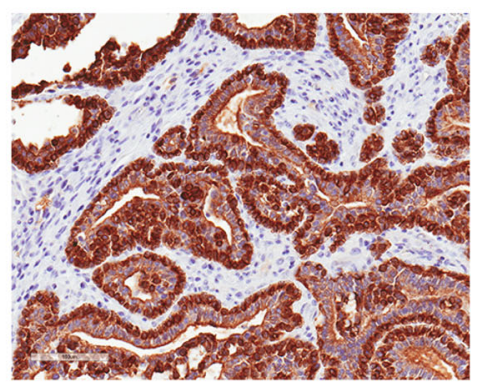

Fig. 2 Nf1 deficiency induces $\mathrm{ER}^{+} / \mathrm{PR}^{+}$mammary tumors. We observed mammary tumors with diverse mammary histology in G0 - G2 animals. a Nf1 females and $\mathbf{b}$ Nf1 males developed mammary adenocarcinomas with mixed histology including acinar, cystic, papillary, ductal, and solid features. All images were taken at 100X magnification. c Immunostaining of Nf1 mammary tumors (rNf1 \#6) for estrogen receptor, progesterone receptor, HER2, Ki67, and pan-cytokeratin (AE1/AE3). All immunostaining images were taken at 200X magnification

and cystic, acinar, and solid (Fig. 2a, right panel). This diverse mammary histology was observed in G0, G1, and G2 animals. In addition, we observed mixed histopathology in the mammary adenocarcinomas from male $N f 1$ rats (Fig. $2 b$ ). The level of tumor burden in mammary pads of several animals was substantial, as can be observed in rNf1 \#413 (Supplementary Table 3). As shown in Supplementary Fig. 2, one mammary gland from rNf1 \#413 contained 6 separate mammary tumors ( 2 additional larger tumors were separated for histopathology). Immunostaining of several tumors revealed that the Nf1 mammary tumors were positive for estrogen receptor, progesterone receptor, and HER2/ Neu receptor and highly proliferative based on Ki67 staining (Fig. 2c). We compared proliferation in the $N f 1^{I F}$ and $N f 1^{P S}$ tumors with Ki67 staining (Supplementary Fig. 3). Even though we observed diversity in proliferation among tumors, quantitative image analysis found no statistically significant difference. The results 
a Tumor Onset

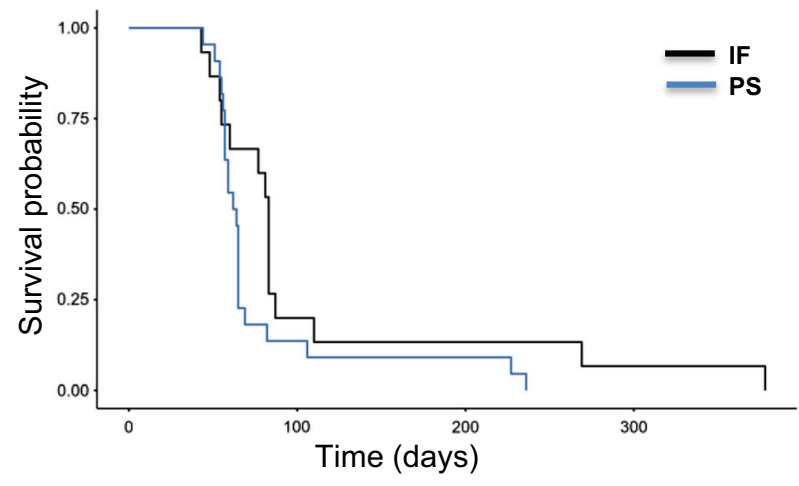

\section{Overall Survival}

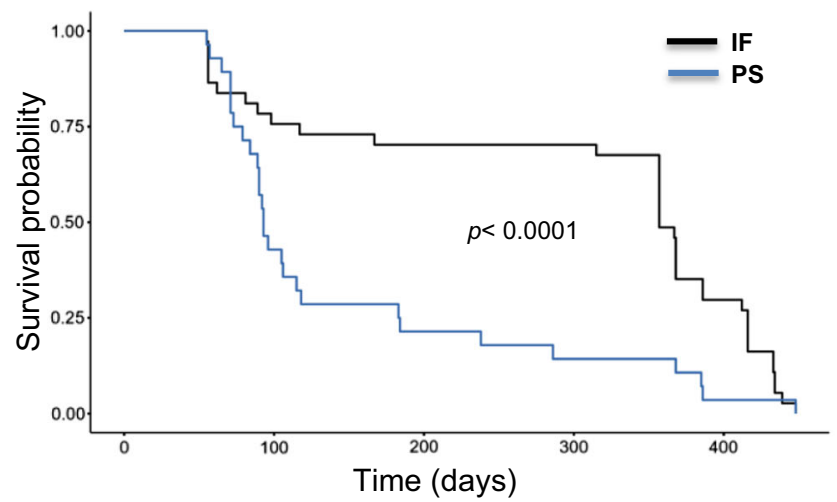

e Overall Survival $N f 1^{I F-57}$ em2 vs. em3

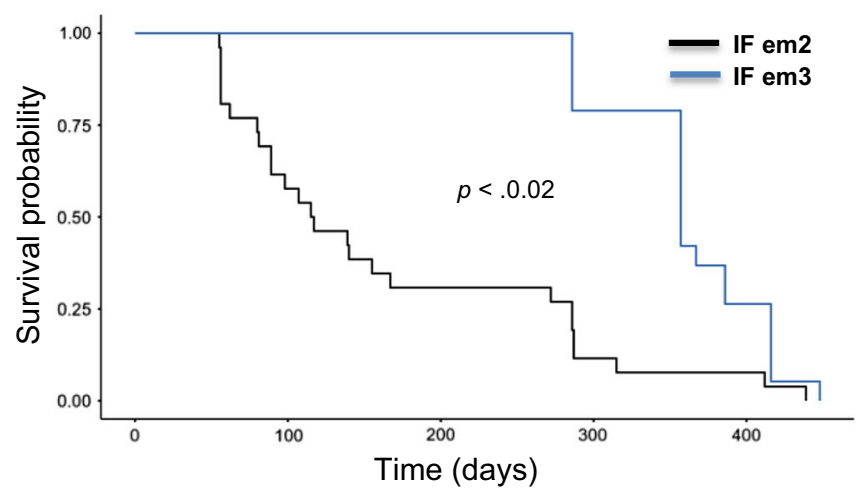

\section{b Disease-specific survival}

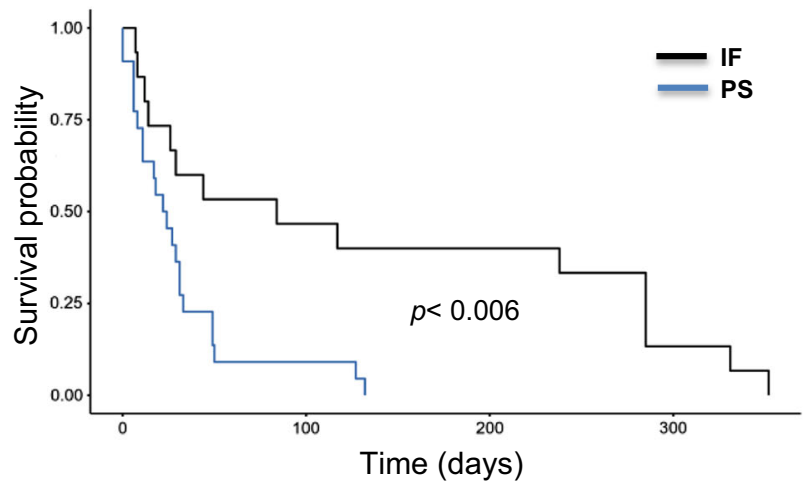

d Tumor Burden em3 IF vs. PS

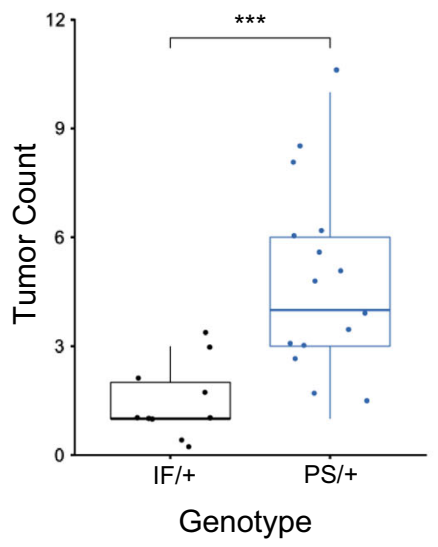

f Tumor Onset em3 IF vs. PS

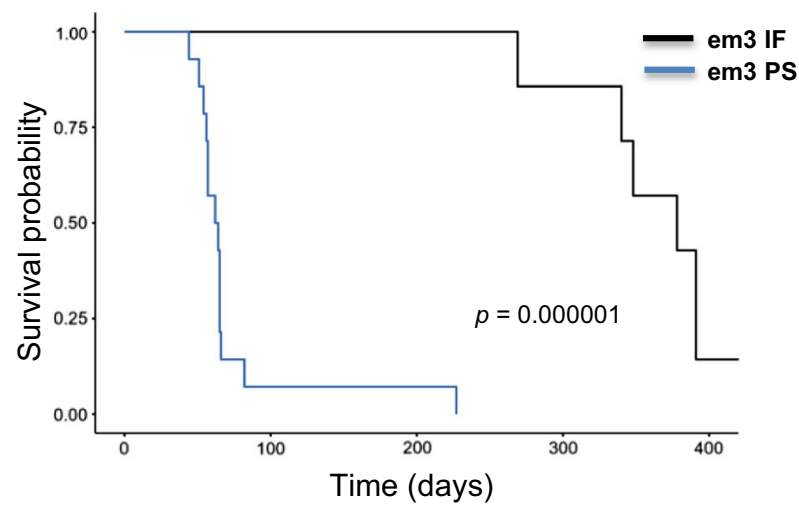

Fig. 3 Survival analysis of $N f 1^{I F}$ vs. $N f f^{P S}$ reveals distinct effects of $N f 1$ deficiency on tumor onset and survival. Survival analysis was performed

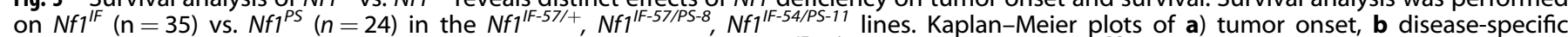
survival, and c overall survival are shown. d Tumor burden of em3-Nf1 ${ }^{1 F-57 /+}$ compared to em3-Nf ${ }^{P S-8}$ rats. e Overall survival analysis of em2

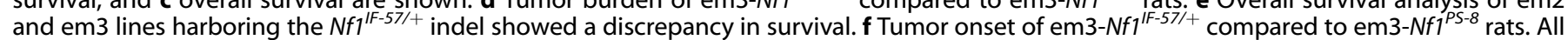
animals in this study died of mammary tumor progression

demonstrate the impact of $N f 1$ loss of function on mammary tumor initiation in both male and female hormonal environments.

Nf1 premature stop indels result in more aggressive initiation and tumor progression

To evaluate how in-frame versus premature $N f 1$ indels affect disease burden and survival, we examined the overall survival of $\mathrm{G} 1$ heterozygous females. We performed a Kaplan-Meier analysis on overall survival of $N f 1^{I F}$ ( $\left.n=35\right)$ compared to $N f 1^{P S}(n=24)$ in three rat lines $\left(N f 1^{I F-57 /+}, N f 1^{1 F-57 / P S-8}, N f 1^{I F-54 / P S-11}\right)$. Survival analysis of tumor onset demonstrated that there was no statistical difference between the time of onset in premature stop compared to in-frame indels (Fig. 3a). Conversely, analysis of disease-specific survival $(p<0.006)$ and overall survival $(p<0.0001)$ revealed that $N f 1^{P S}$ animals died due to tumor burden significantly faster than $N f 1^{\text {IF }}$ animals (Fig. 3b, c). These data were influenced by a 
a

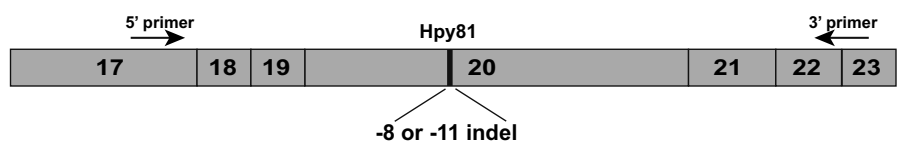

b
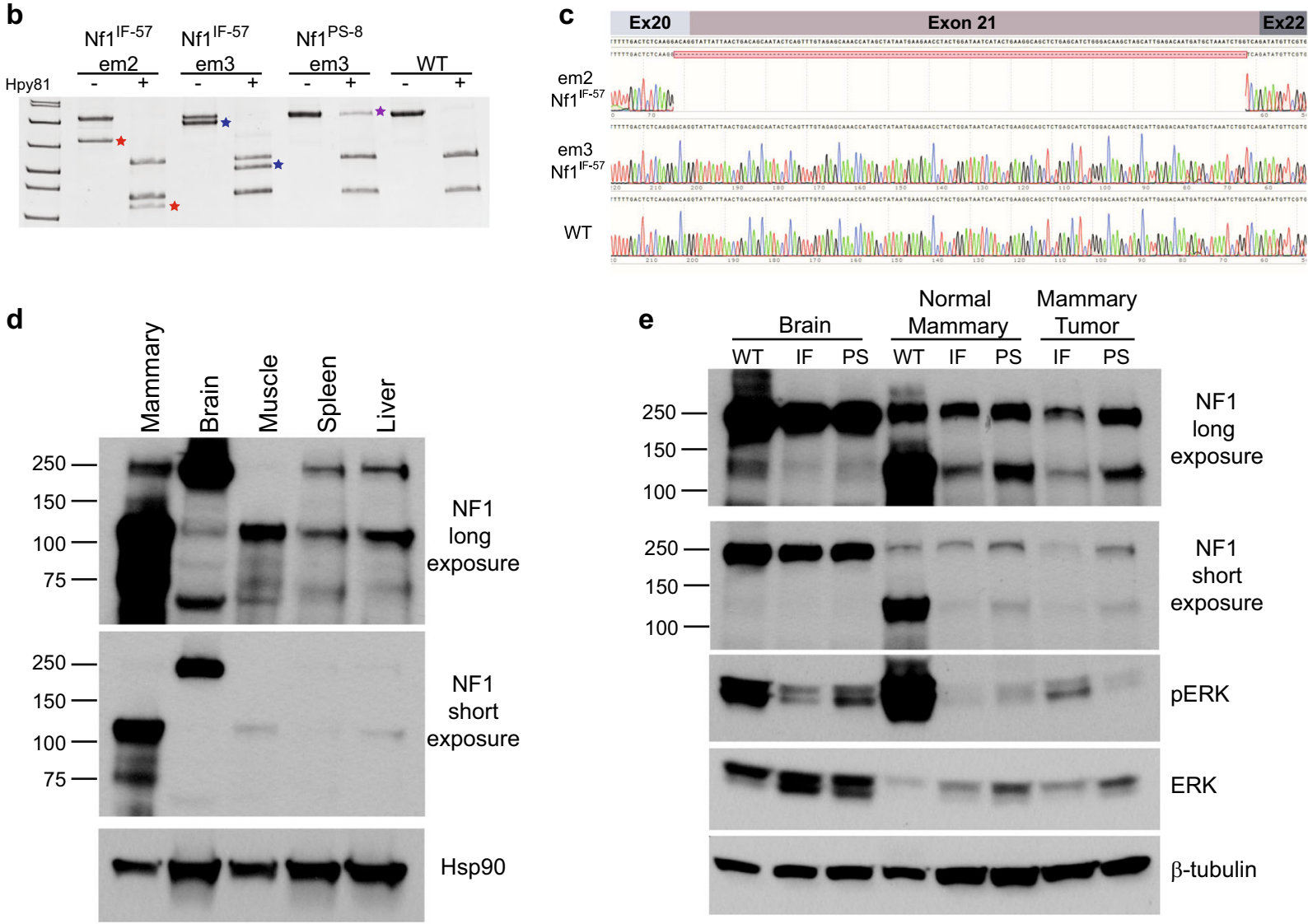

$\mathbf{f}$

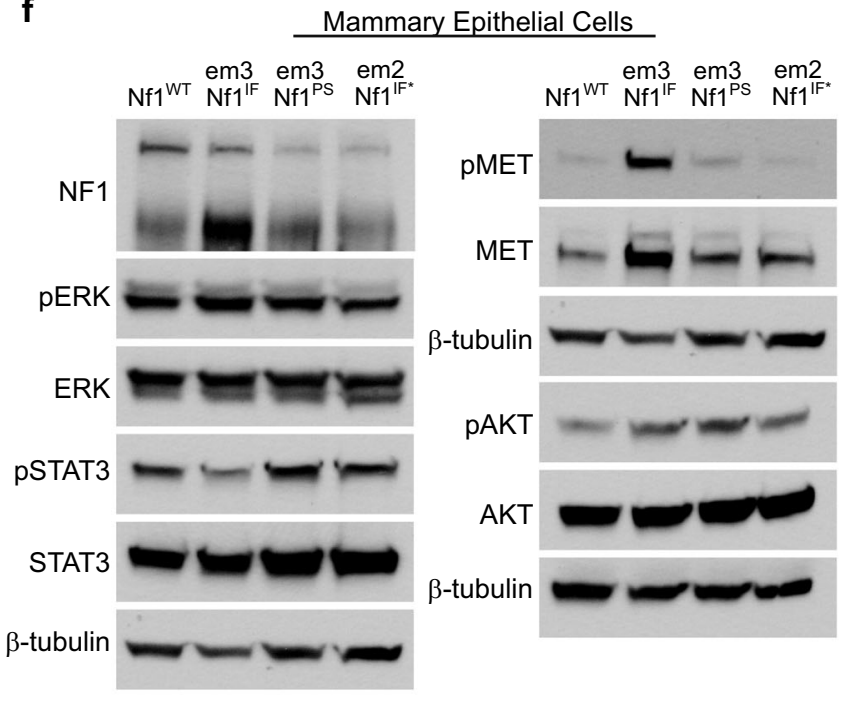

g

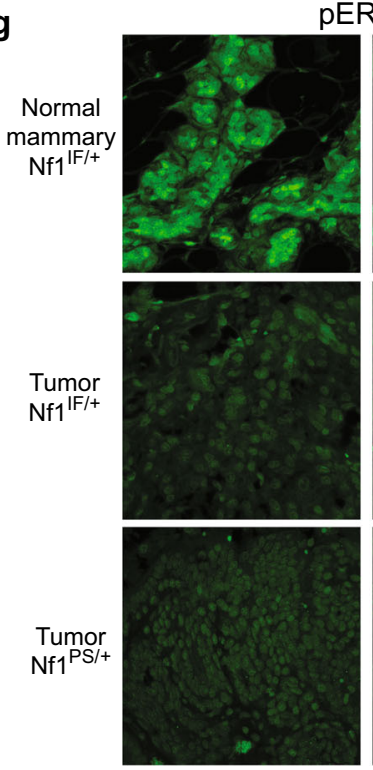

ERK
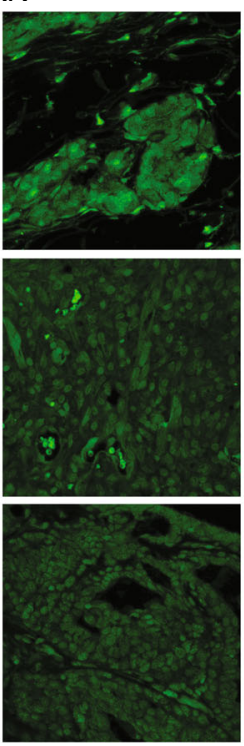

significant increase in tumors observed in $N f 1^{P S}$ animals. As shown in Fig. $3 \mathrm{~d}, N f 1^{P S}$ animals had an average of $3 \mathrm{X}$ more tumors than $N f 1^{l F}$ animals ( $\left.p=0.00006,95 \% \mathrm{Cl}[1.82,5.41]\right)$. Notably, all animals in this study died of mammary tumor progression. We compared the overall survival of two $N f 7^{1 F-57}$ lines that were derived from separate founders (em2 and em3). Interestingly, we observed that the em2-Nf ${ }^{1 F-57}$ rats had a significantly shorter survival than em3$N f 1^{I F-57}$ rats, even though these $N f 1$ lines had identical in-frame deletions $(p<0.02$; Fig. 3e). The potential reason for this discrepancy in survival is described below. 
Fig. 4 Analysis of $N f 1^{I F}$ and $N f 1^{P S}$ mRNA and protein isoforms. a Schematic of RT-PCR analysis of Nf1 exons 17-23. b RT-PCR products were digested with Hpy81 and separated by PAGE to differentiate $N f 7^{1 F}$ and $N f 1^{P S}$ mRNA. Unique bands were observed in the em2-Nf1 $1^{I F-57}$ and em3$N f^{I F-57}$ REF CDNA (noted by red and blue stars). The PS mRNA that is not digested due to the loss of the Hpy 81 site is noted (purple star). $c$ Sequence analysis of em2-Nf $1^{\mid F-57}$ and em3-Nf $1^{\mid F-57}$ REF CDNA. d, e Immunoblot analysis showing d tissue-specific expression of rat neurofibromin isoforms and e differential expression of the neurofibromin 250 and $125 \mathrm{kD}$ bands in matched brain, normal mammary tissue, and mammary tumor at both a long (top) and short (bottom) exposure. f Western blot analysis of neurofibromin, ERK, STAT3, MET, and AKT phosphorylation and expression in MECs derived from normal Nf1 rat mammary pads. g Representative immunofluorescence of pERK1/2 in normal mammary tissue and mammary adenocarcinomas. Images are shown at $\times 600$

Distinct neurofibromin isoform is expressed in mammary tissue To understand how Nf1 deficiency via mutation of the GRD domain affects neurofibromin expression and activity, we first examined the $N f 1$ isoforms that are expressed in distinct $N f 1^{I F}$ and $N f 1^{P S}$ lines. mRNA was isolated from day 13.5 rat embryos fibroblasts (REFs) and RT-PCR was performed using primers in exon 17 and exons 22-23 (Fig. 4a). To differentiate RT-PCR products derived from the WT, IF, and PS alleles, we performed restriction digests with the Hpy81 endonuclease (Fig. 4b). As demonstrated in Fig. 1d, e, alleles harboring premature stop indels have lost the Hpy81 restriction site at the $5^{\prime}$ CRISPR site. RT-PCR of WT REFs resulted in a strong band at $826 \mathrm{bp}$ that created two bands at $390 \mathrm{bp}$ and $368 \mathrm{bp}$ with Hpy81 digestion. RT-PCR and Hpy81 digestion of the $N f 1^{P S-8}$ fibroblasts resulted in $N 7^{W T}$ bands and an undigested band at $745 \mathrm{bp}$. Expression of the WT allele was observed in each of the Nf1 in-frame and premature stop REF lines. We then compared the em2- Nf $1^{I F-5}$ and em3-Nf1 ${ }^{I F-57}$ lines that had distinct survival curves (Fig. 3e). Even though genotyping confirmed an identical 57 bp deletion in exon 20 in both lines, we observed distinct Nf1 mRNA isoform expression patterns (Fig. 4b, notated with *). To determine the difference in the $N f 1^{\mid F-57}$ mRNA isoforms, we cloned and sequenced each of the products. Overall, we sequenced seventeen clones where the majority contained the predicted $N f 1^{I F-57}$ and $N f 1^{W T}$ isoforms, but we identified 3 unique clones harboring exon 21 deletions or extended exon 20 deletions resulting in Nf1 premature stops. For example, one mRNA isoform contained the $57 \mathrm{bp}$ in-frame deletion in exon 20 and an additional $140 \mathrm{bp}$ deletion in exon 21 (Fig. 4c). This exon 21 deletion mRNA was exclusive to the em2-Nf1 ${ }^{1 F-57}$ line and resulted in a premature stop that would impact the GRD domain and other downstream functional domains. Because our initial analysis of tumor onset (Fig. 3a) included the em2-Nf1 ${ }^{\mid F-57}$ animals as "IF", we repeated our statistical analysis of tumor onset in em3-Nf1F-57 versus em3-Nf1 ${ }^{P S-8}$ animals. As shown in Fig. 3f, the tumor onset was significantly earlier in $N f 7^{P S}$ rats compared $N f 1^{I F}$ animals. These results substantiate the requirement of a functional GRD domain within NF1 to suppress tumor progression.

Several unique isoforms have been characterized in the Nf1 gene with the main isoform identified at $250 \mathrm{kD}$; however, the majority of these studies have been performed in brain, neuronal, and muscle tissues. ${ }^{38-40}$ To our knowledge, neurofibromin expression has not been previously evaluated in the mammary glands of humans or rodents. To understand the effect of Nf1 deficiency in the mammary gland, we first performed Western blot analysis of neurofibromin in the normal mammary, brain, muscle, spleen, and liver of adult rats and observed distinct isoform expression patterns among the tissue types (Fig. 4d). Even though the established $250 \mathrm{kD}$ neurofibromin isoform is highly expressed in brain tissue, its expression was significantly less in other tissues, including the mammary gland. In the mammary gland, the predominant neurofibromin isoform was observed at $125 \mathrm{kD}$. This $125 \mathrm{kD}$ isoform was also observed in the muscle, spleen, and liver, yet minimally expressed in the brain. In Fig. 4e, we compared neurofibromin expression in the brain, normal mammary glands, and mammary adenocarcinomas from $N f 1^{W T}, N f 1^{I F}$, and $N f 1^{P S}$ rats. In the brain, the $250 \mathrm{kD}$ isoform was highly and equally expressed in the $N f 1^{W T}, N f 1^{I F}$, and $N f 1^{P S}$ animals. In the normal mammary gland, there was no difference in expression of the $250 \mathrm{kD}$ isoform between the $N f 1^{W T}, N f 1^{I F}$, and $N f 1^{P S}$ animals, however there was a substantial decrease in expression of the $125 \mathrm{kD}$ isoform in both the $N f 1^{I F}$ and $N f 1^{P S}$ mammary glands compared to WT mammary glands. In mammary adenocarcinomas, $125 \mathrm{kD}$ isoform was further decreased in both the $N f 1^{I F}$ and $N f 7^{P S}$ tumors and there appeared to be a slight loss of the $250 \mathrm{kD}$ isoform in the $N f 1^{\prime F}$ mammary tumor. Overall these results demonstrate the distinct neurofibromin isoform tissue preferences that are present and the inverse relationship of the $250 \mathrm{kD}$ vs. $125 \mathrm{kD}$ isoforms in the brain and mammary tissues.

To determine the effect of Nf1 deficiency on RAS signaling, we examined ERK staining in normal mammary tissue, mammary epithelial cells, and mammary tumors. In normal mammary glands we observed a very strong $\mathrm{pERK}$ signal that was unexpectedly lower in the $N f 1^{I F}$ and $N f 1^{P S}$ normal mammary and tumor tissue (Fig. 4e). In normal brain tissue where the $250 \mathrm{kD}$ neurofibromin isoform is predominantly expressed, total ERK was increased in the $N f 1^{I F}$ and $N f 1^{P S}$ brains compared to $N f 1^{W T}$, yet pERK was strongest in the $N f 1^{W T}$ tissue (Fig. 4e). Given that the mammary pad is composed of primarily stromal cells (i.e., adipocytes, fibroblasts, immune cells), we wanted to evaluate the effect of Nf1 deficiency specifically in the mammary epithelium. We optimized a procedure to isolate mammary epithelial cells (MECs) from normal rat mammary fat pads and examined $N f 1$ expression and signaling in $N f 1^{W T}, N f 1^{I F}$, and $N f 7^{P S}$ MECs (Fig. 4f). In the MECs, we observed distinct differences in neurofibromin expression and signaling compared to the whole mammary tissue. In the $N f 1^{W T}$ MECs, both the $250 \mathrm{kD}$ and $125 \mathrm{kD}$ isoforms were expressed and in the em3$N f 1^{I F}$ MECs, expression of the $125 \mathrm{kD}$ neurofibromin isoform was moderately increased. In contrast, in $N f 1^{P S}$ and em2-Nf1 $1^{I F^{*}}$ (which express the additional exon 21 mRNA deletion and mimic the $N f 1^{P S}$ ) MECs, neurofibromin $250 \mathrm{kD}$ expression was decreased. This decrease in neurofibromin expression resulted in no change in total ERK or pERK compared to $N f 1^{W T}$ (Fig. 4f). Next, we assessed pERK subcellular localization using immunofluorescence and observed cytoplasmic pERK in the normal mammary pad (Fig. 4g; Supplementary Fig. 4). In contrast, we observed predominantly nuclear pERK in tumors from $N f 1^{I F}$ and $N f 1^{P S}$ animals (Fig. 4g; Supplementary Fig. 4a). When we compared pERK staining in the tumor periphery compared to the interior, we observed a moderate increase in ERK activity at the tumor periphery (Supplementary Fig. 4b). We also examined AKT, MET, and STAT3 which we and others have shown to be critical signaling pathways involved in the progression of NF1-related sarcomas. ${ }^{41,42}$ For the MET receptor we observed an increase in both activated (phosphorylated MET) and total MET in only the $N f 1^{I F}$ MECs (Fig. $4 \mathrm{f}$ ), yet immunostaining revealed an increase in pMET in both $\mathrm{Nf} 1^{I F}$ and $N f 1^{P S}$ tumors compared to normal mammary tissue (Supplementary Fig. 5a). There was no change in AKT expression or phosphorylation in the MECs or tumors, yet we observed an increase in both pSTAT3 expression and nuclear localization in both the $N f 1^{I F}$ and $N f 1^{P S}$ MECs and tumors (Fig. 4f; Supplementary Fig. 5b). These results indicate that several of the RAS linked signaling pathways altered in other NF-related cancers are altered in Nf1-mediated mammary tumor progression. 

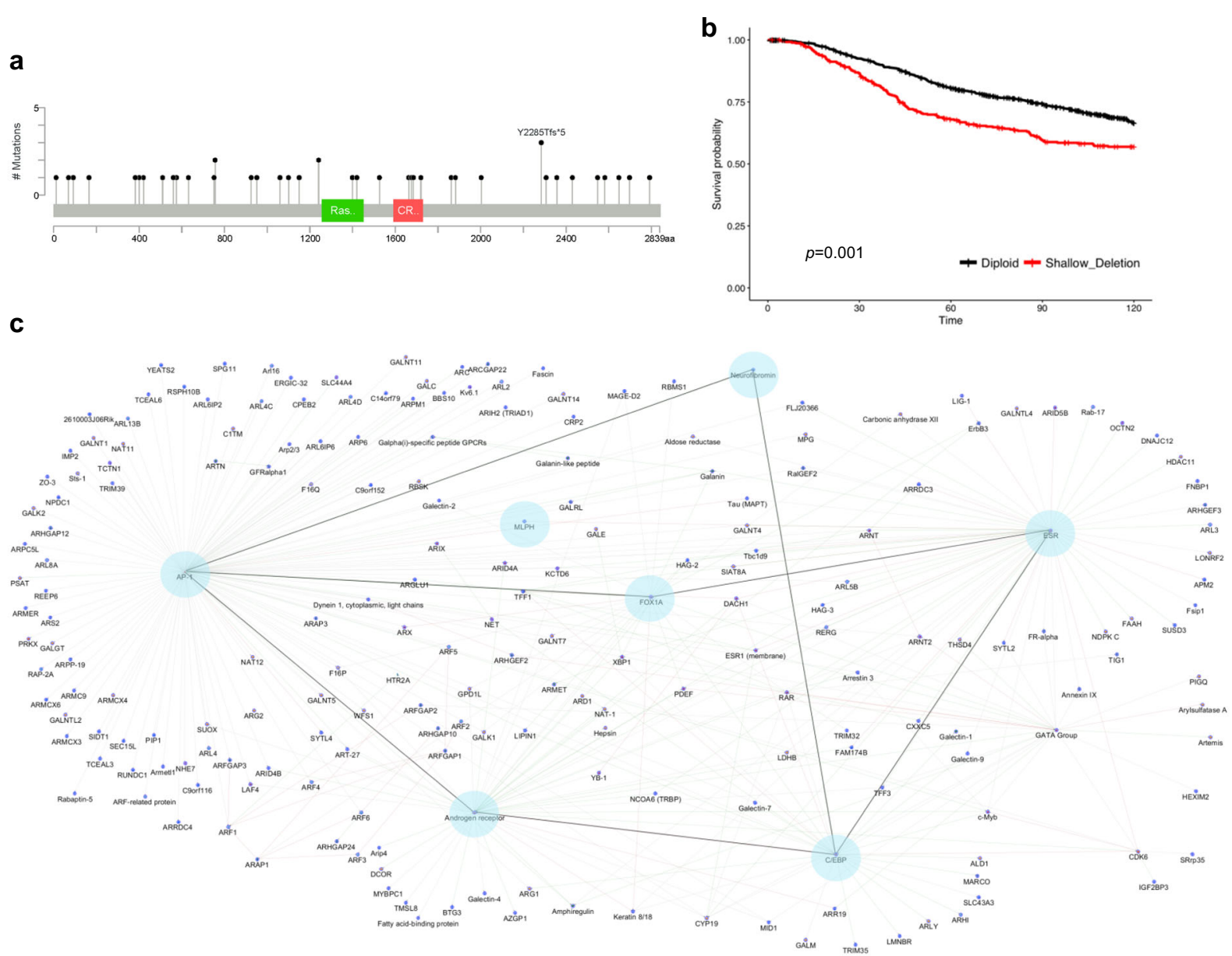

d

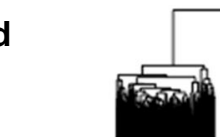

ER STATUS | ||

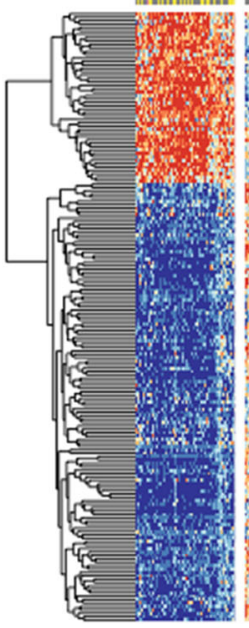

Cluster

1
NF1 STATUS $\||\|||||||||| \mid$
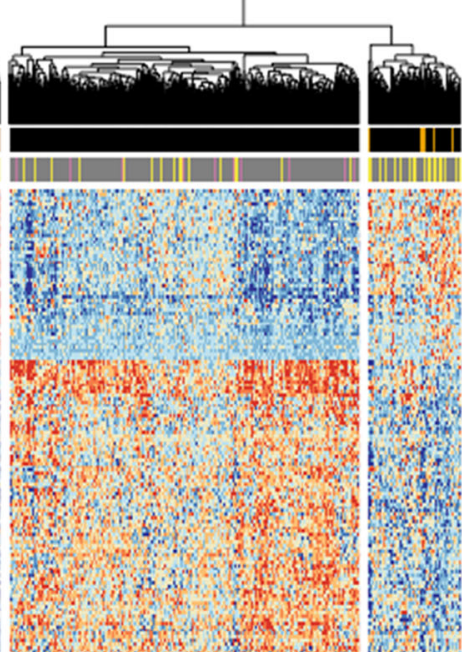

8
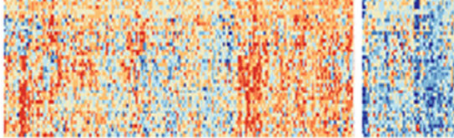

Cluster

Cluster

3

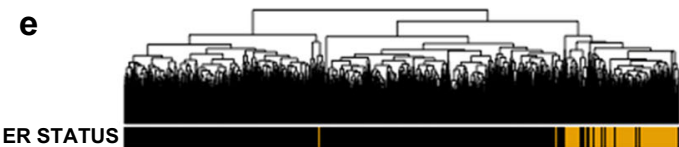

NF1 STATUS $\|$
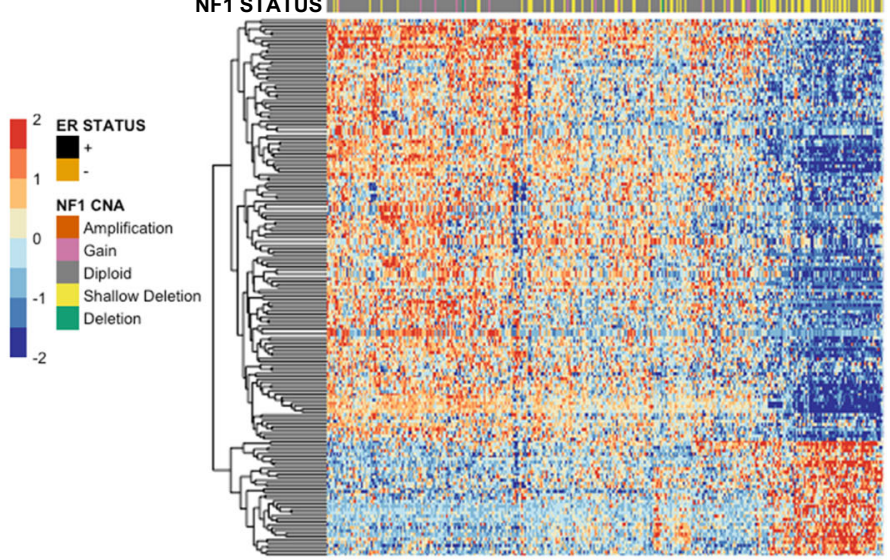

Fig. 5 NF1 shallow deletions are frequently present in sporadic breast cancers and associate with ESR1 and FOX1A. a Truncating mutations were detected throughout NF1 in METABRIC patients (cBioportal). b Survival analysis of breast cancer patient survival comparing NF1 diploid and shallow deletion copy number status. c Network visualization of WGCNA module genes associated with NF1 shallow deletion copy number status. d Heatmap of METABRIC gene expression data of WGCNA module genes correlated with NF1 copy number and ER status. e Heatmap of METABRIC gene expression data of WGCNA module genes associated with RAS activation signatures 
NF1 is commonly deleted in human sporadic breast cancer and correlates with ER networks

Even though it is known that neurofibromatosis patients have a significantly increased breast cancer risk and that NF1 is mutated in sporadic breast cancers, the impact of NF1 deficiency and RAS deregulation in sporadic breast cancer is often overlooked. To interrogate the impact of NF1 loss in breast cancer, we performed an analysis of the METABRIC breast cancer data set which contained 2,051 patients with clinical annotation including CNV and SNP genotypes. ${ }^{33}$ In this cohort, there were 43 patients (2.1\%) with truncating mutations, including frameshift deletions or insertions, nonsense mutations, and splice site alterations (Fig. $5 \mathrm{a})$. Notably, these mutations occurred throughout the NF1 gene similar to the mutation diversity observed in NF patients. After removing patients with NF1 SNP mutations, we analyzed NF1 copy number alterations (CNAs) and identified CNAs in $32.9 \%$ of patients, with $24.5 \%$ having NF1 shallow deletions (defined as potential heterozygous deletions). To determine the effect of NF1 shallow deletions on survival, we conducted a survival analysis using the Cox proportional hazards model adjusting for ER status and age at diagnosis. We found that patients with an NF1 shallow deletion had significantly higher tumor grade, stage, and size (Supplementary Table 4). They were also more likely to have basallike breast cancer $(p<0.0001)$ and less likely to have luminal $A$ breast cancers $(p<0.0001)$. Moreover, patients with an NF1 shallow deletion had a hazard ratio of $1.4(p=0.001)$ relative to diploid NF1 status for 10 year survival (Fig. 5b, Supplemental Table 4).

To identify gene expression networks that correlate with NF1 copy number status, we utilized weighted gene co-expression network analysis (WGCNA). ${ }^{43}$ From the METABRIC data set, expression data were analyzed for patients with available copy number and gene expression data $(n=1427)$. Since NF1 is in close proximity to HER2 on chromosome 17 and is often co-amplified, we removed HER2-amplified patients from this analysis. From the 18,049 genes in the METABRIC data set, 2,218 were placed in 12 co-expression modules. Modules contained between 30 and 500 genes, with an average module size of 200 genes. Interestingly, one module (179 genes) associated with NF1 shallow deletion copy number status contained several genes that are considerably important in both ER+ breast cancers and endocrine resistance, including ESR1 (Fig. 5c). Hub genes for this module, or genes with high connectivity, included FOXA1 and MLPH. FOXA1, a Forkhead family transcription factor, regulates ER binding and transcriptional activity. ${ }^{44}$ FOXA1 expression correlates with luminal subtype A breast cancer and is a significant predictor of cancer-specific survival in ER+ tumors. ${ }^{45} \mathrm{MLPH}$ expression is also associated with longer survival in breast cancer. ${ }^{46}$ Further network visualization of genes within this ER-associated co-expression network revealed additional connections with $A P 1, A R$ (androgen receptor) and C/ $E B P a$ (CCAAT/enhancer binding protein). AP1 is also known as the transcription factor JUN. RAS/ERK signaling is known to regulate JUN at the transcriptional and post-translational (phosphorylation) level and can modify lysine acetylation in JUN DNA binding regions. ${ }^{47}$ Recent studies have demonstrated that $A R$ is expressed in $77 \%$ of breast cancers $(88 \% \mathrm{ER}+, 59 \% \mathrm{HER} 2+, 32 \% \mathrm{TNBC})^{48}$ and is involved in endocrine resistance in ER+ breast cancers. ${ }^{49,50}$ Although there are studies demonstrating interaction of $C / E B P a$ at $E R$ transcriptional binding sites, the consequence of $C / E B P a$ activity in breast cancer is unclear.

Unsupervised clustering of genes from this WGCNA module with METABRIC clinical data revealed a strong association of NF1 shallow deletions in both ER+ and ER- tumor subsets. This analysis distinguished three modules associated with NF1 copy number status, with one module comprised of 181 genes associated specifically with NF1 shallow deletions (Fig. $5 \mathrm{~d}$ ). The heatmap in Fig. $5 d$ revealed three clusters associated with NF1 and ER status:
Cluster 1 (primarily ER-), Cluster 2 (ER+), and Cluster 3 (primarily ER + ). The odds of Cluster 1 patients having an NF1 shallow deletion (versus another mutation) is 2.01 times higher than Cluster 3 and 5.55 times higher than Cluster $2(p=0.0002$ and $p<0.0001 ; 95 \%$ $\mathrm{Cl}[1.39,2.89]$ and $4.08,7.63]$, respectively). Whereas, the odds of Cluster 3 having a shallow deletion is 2.76 times higher than Cluster 2 ( $p<0.0001 ; 95 \% \mathrm{Cl}[1.99,3.84])$. To understand if NF1 shallow deletions correlate with deregulated RAS signaling, we employed expression signatures developed from KRAS-mutant cancers and performed a secondary WGCNA analysis and unsupervised clustering with 788 genes related to ER, RAS and KRAS signaling pathways (Fig. 5e). These results validate the presence of RAS activation in NF1-related breast cancers. Moreover, our WGCNA analyses revealed novel connections between NF1 deficiency, RAS signaling, and ER signaling.

\section{Nf1-deficient tumors are estrogen-dependent}

To examine whether our Nf1-deficient rat breast cancer model was estrogen dependent, we performed ovariectomies on rats containing at least one tumor greater than $1500 \mathrm{~mm}^{3}$. Ovariectomies were performed on 7 rats with multiple tumors from three distinct Nf1 lines (Fig. 6a-c). Upon ovary removal, tumor size diminished at a rapid pace (mean reduction per day $=4.7 \%$ per day $(p<0.0001 ; 95 \% \mathrm{Cl}[3.8,5.5])$. Tumor reduction was observed in all of the tumors regardless of Nf1 status (in-frame vs. premature stop indels) and the mean total percent reduction in tumor volume was $97.8 \%$ ( $p<0.0001 ; 95 \% \mathrm{Cl}[94.1,99.8])$ (Fig. 6c). ER is tightly regulated through numerous post-translational modifications including phosphorylation, acetylation, methylation, sumoylation, and ubiquitination. Previously it has been demonstrated that ERK can phosphorylate ERa at S104/106 and S118, where S118 phosphorylation facilitates ERa interactions with coactivators. ${ }^{51,52}$ We examined ERa expression and S118 phosphorylation in MECs and observed no change in total ER, but pERa was increased in both $N f 1^{P S}$ and $N f 1^{I F *}$ MECs (Fig. 6d). This pattern matched the level of decrease in neurofibromin expression observed in Fig. 4f. When we examined $p$-ERa (S118) in normal mammary tissue from an $N f 1^{W T}$ rat, we observed weak to moderate staining in the mammary epithelial cells (Fig. 6e). In contrast, mammary adenocarcinomas from both $N f 1^{I F}$ and $N f 1^{P S}$ animals had extremely intense ERa pS118 staining. Strong p-ERa was also observed in $N f 1^{I F}$ mammary tissue adjacent to a tumor in comparison to the $N f 1^{W T}$ mammary tissue. These results confirm estrogen-dependency in the rat Nf1-deficient breast cancer model and support the biological significance of the NF1-ER networks that were identified by WGCNA.

\section{DISCUSSION}

The RAS signaling pathway is intricately involved in the initiation and progression of numerous cancer types; however, the mechanisms by which RAS is deregulated in breast cancer are unclear. Here we present data that illuminates the role that NF1 plays in breast cancer and a novel model for interrogating the interaction between NF1 and ER in both sporadic and NF-related breast cancer. Even though NF patients are predisposed to variety of cancers, breast cancer risk was not confirmed until recently. ${ }^{15-17}$ NF1 was first implicated in sporadic breast cancer by the initial comprehensive genome breast cancer studies where NF1 mutations were identified in $2-4 \%$ of breast cancers. ${ }^{18,19}$ Our analysis of the extensive METABRIC data set verified the NF1 mutation frequency, but also discovered NF1 shallow deletions in $25 \%$ of sporadic breast cancers. The analysis also revealed NF1 shallow deletions correlate with higher tumor grade, tumor size, and the aggressive, basal subtype. Most importantly, NF1 shallow deletions correlate with poor outcome within the first 10 years. Thus, our work strengthens the conclusion drawn from the TCGA breast 
a

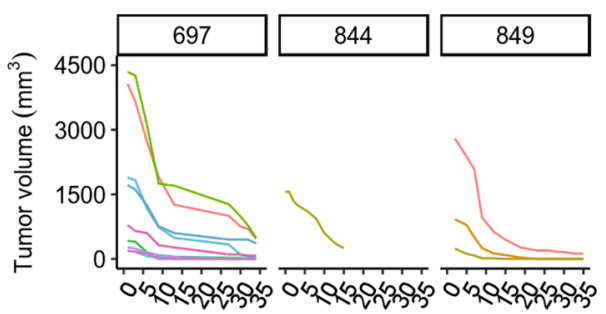

b

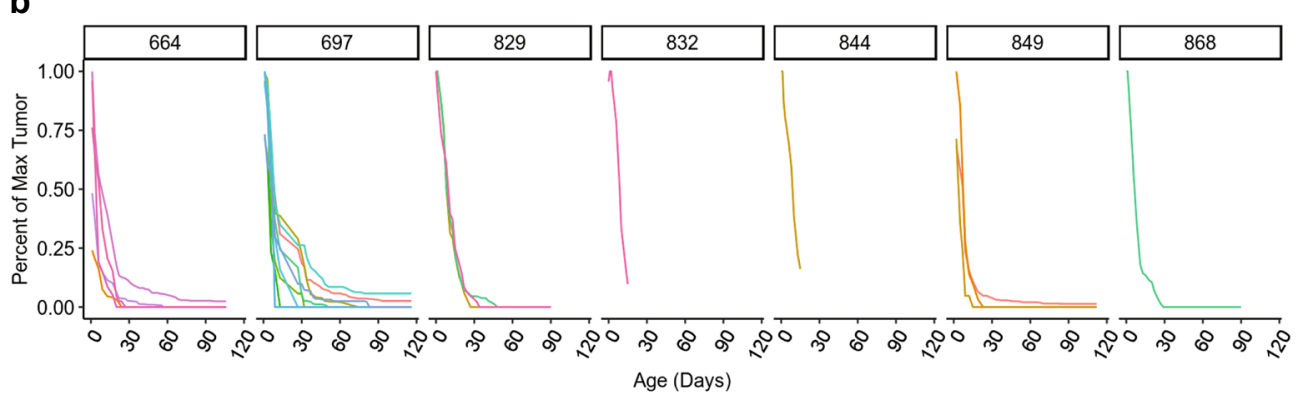

C

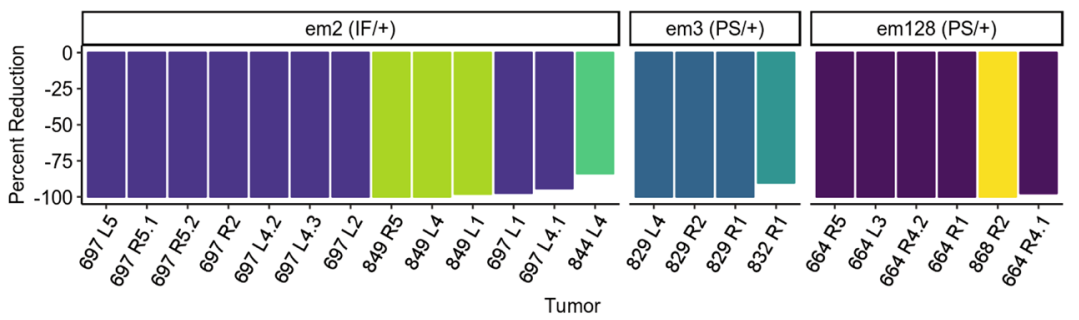

d

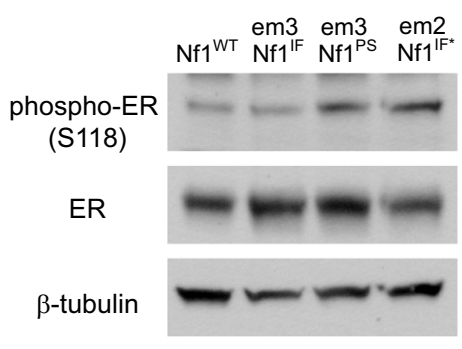

e

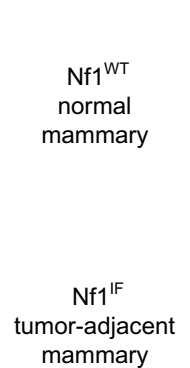

mammary

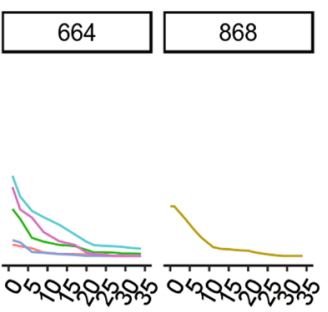

Age (Days)

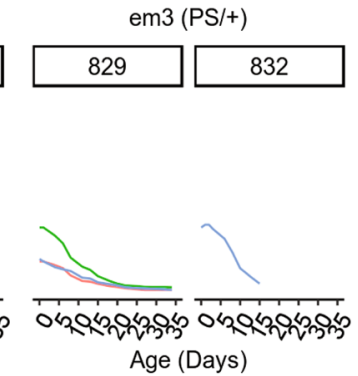

em3 (PS/+)
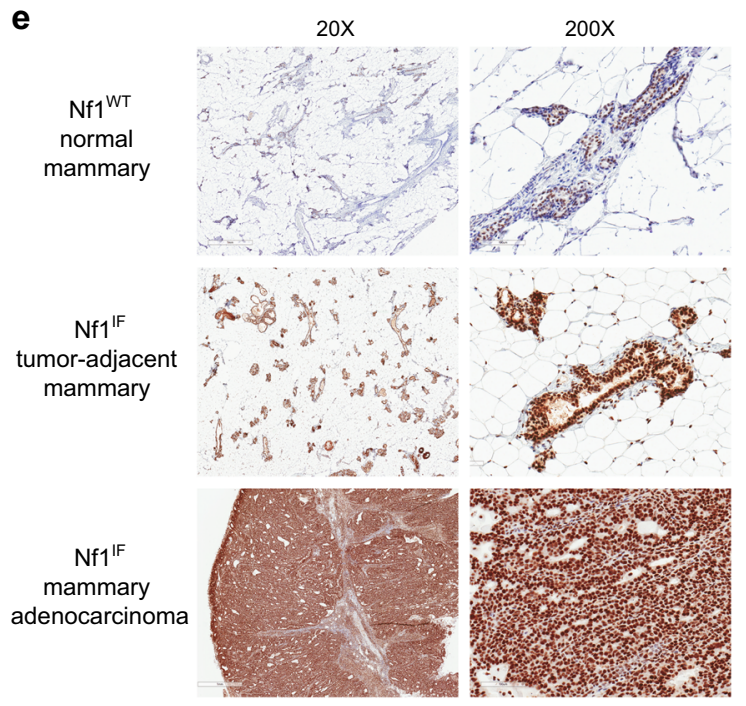

$\mathrm{Nf} 1^{\text {PS }}$

mammary

adenocarcinoma
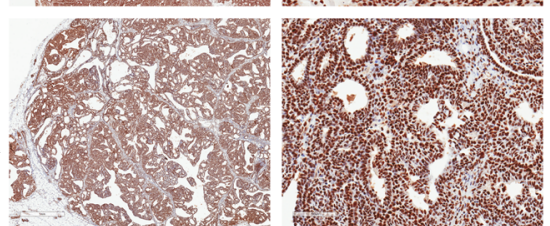

Fig. 6 Estrogen depletion via ovariectomy results in rapid tumor regression in Nf1-deficient tumors. a Tumor volume was plotted after ovariectomy (Day 0) for individual $N f 1^{I F}$ and $N f 1^{P S}$ rats. b Percent tumor change over time of multiple tumors in individual $N f 1^{\mathrm{IF}}$ and $N f 1^{\mathrm{PS}}$ rats after ovariectomy (Day 0). c The percent reduction in tumor size for all tumors from $3 \mathrm{Nf1}$ rat lines is shown. Distinct colors are used to represent individual rats and the mammary pad location of each tumor is notated. Note that several rats had multiple measurable tumors within an individual mammary pad. d Immunoblot analysis of phospho-ER (S118) and ER expression in MECs derived from normal Nf1 rat mammary pads. e Immunostaining of phospho-ER (S118) in normal mammary tissue and mammary adenocarcinomas from Nf ${ }^{W T}, N f 1^{I F}$ and $N f 1^{P S}$ rats 
cancer data set and the role of NF1 as a bona-fide driver. ${ }^{20}$ In addition to NF1, the RasGAP gene RASAL2 is altered in sporadic breast cancer. McLaughlin et al. demonstrated that RASAL2 expression is substantially decreased in luminal $B$ breast cancers and low RASAL2 expression correlates with metastasis and poor survival. ${ }^{53}$ Our analysis extends these findings towards the basal subtype which exhibits a disproportionately increased number of NF1 shallow deletions. Taken together, these studies indicate that loss of RAS suppression may be more important in breast cancer than RAS activation through mutation or amplification.

Our WGCNA analysis revealed an unknown network between $N F 1, E S R 1$, and RAS signaling in breast cancer. In our Nf1 tumor model, we observed strong ER (S118) phosphorylation indicating a role for indirect effects of Nf1 deficiency through ERK phosphorylation of the S118 motif. However, recent findings demonstrate direct NF1-ER binding that is mediated by the ER ligand binding domain and NF1 leucine-rich regions. ${ }^{54}$ These exciting results indicate that NF1 acts like a classic ER corepressor and that corepressor activity requires NF1 GAP activity and the Leu-rich motif. Our results complement these findings and strongly suggest that NF1 deficiency directly and indirectly impacts ER signaling. Interrogating the mechanisms by which NF1 interacts with ER will require a careful analysis of both transcriptional and signaling interactions in various hormonal environments. Neurofibromin is a large protein and there is a limited knowledge of neurofibromin protein-protein interactions besides the RAS interactions at the GRD domain. Even though neurofibromin may directly interact with genes involved identified in the NF1-ER network, indirect NF1-RAS-ER signaling interactions are likely since RAS signaling regulates JUN (AP1) at the transcriptional and posttranslational level. ${ }^{47}$ It is also possible that direct neurofibromin-ER interactions are isoform dependent. Using our Nf1 rat model, human breast cancer lines, and patient-derived xenograft models, we will be able to interrogate how the presence and absence of estrogen impacts direct and indirect NF1-ER signaling.

Together, our CRISPR rat Nf1 model and our WGCNA analysis of human breast cancer confirms that NF1 is intricately connected to ER networks, including several genes that have been directly linked to endocrine resistance (i.e., FOXA1 and $A R$ ). It is interesting to note that the rate of tumor regression in the $N f 1$ tumors after estrogen ablation was remarkably rapid and included all Nf1 tumors regardless of mutation (PS or IF). These data suggest that our Nf1-deficient tumors are universally estrogen-dependent. The WGCNA data indicate more distinct roles for NF1 in both estrogen positive and negative environments. One potential explanation for these divergent results is that Nf1-deficient tumors are treatment naïve, occurring in younger animals, whereas adult tumors in the METABRIC data set are diverse with respect to treatment and host factors. A recent study showed that NF1 mutations contribute to the genomic evolution towards metastasis and are prevalent in recurrent, ER + breast cancers. ${ }^{55}$ Considering our demonstration that (1) NF1 shallow deletions correlate with poor outcome, (2) network connectivity exists between NF1-ER-FOXA1-AR, and (3) broad response of $N f 1$ tumors to ovariectomy, it is likely that NF1 is a key factor in endocrine resistance.

The $N f 1$ rat model also revealed some unexpected alternative mRNA and protein isoforms that underscore our limited understanding of NF1 expression. For example, we observed two distinct neurofibromin isoforms that are differentially expressed in the brain and mammary gland. Both in-frame and premature stop sequence variants resulted in significantly reduced neurofibromin mammary expression, whereas expression of the $250 \mathrm{kD}$ isoform was largely preserved in the brain regardless of $N f 1$ status. Collectively, these findings suggest that RAS regulation in the breast is distinct from other tissues, and that breast cancer predisposition may be linked to a unique isoform of neurofibromin that is abundantly expressed in mammary tissue.
One issue that vexed us initially was the divergence of survival between two genotype-matched $N f 7^{1 F-57}$ lines. Analysis of the

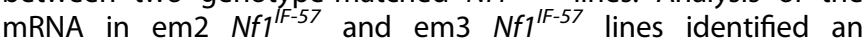
additional deletion in exon 21 in em2 Nf1 ${ }^{I F-57}$. Differential mRNA isoform expression has been observed in breast cancer and may occur through alternate promoter usage, alternate splicing, and alternate $3^{\prime}$ UTR usage. ${ }^{56}$ Importantly, differential mRNA isoform expression has been identified and associated with distinct breast cancer subtypes. ${ }^{56}$ Specific to the NF1 gene, splice variation has also been described. ${ }^{57,58}$ Our data strongly extend these findings as we show that both mutation type (IF and PS) and splice variation lead to more aggressive disease. It is possible that NF1 isoform expression and splice variation instruct tumor development, and may account for variability in genotype-phenotype correlation.

In summary, we have developed a novel Nf1 rat model that is invaluable for interrogating deregulated RAS signaling and ER networks in sporadic and NF-related breast cancer. Moreover, we identified a frequent mechanism for RAS deregulation through NF1 shallow deletion that is present in $25 \%$ of sporadic breast cancers. The correlation of NF1 shallow deletions with poor prognosis and the ER-FOXA1-AR networks indicates that NF1 may be an important prognostic indicator and therapeutic target in endocrine-resistant breast cancers.

\section{METHODS}

CRISPR/sgRNA design and synthesis, and Cas9 mRNA synthesis CRISPR targets were identified in exon 20 of the rat Nf1 gene (NCBI rn5 rat genome) using an online design tool (crispr.mit.edu). A region near the middle of exon 20 was targeted by one guide sequence ( $5^{\prime}$ CRISPR), GCCCTGTCAGTGAACGCAAA (GGG), while a downstream region was targeted by a second guide sequence ( $3^{\prime}$ CRISPR), CGGTCCATAAATCTGCTGAC (AGG). The rn5 rat genome reference sequence and annotation was used for CRISPR target design.

Rat breeding, microinjection into zygotes and blastocyst culture Sprague-Dawley rats were purchased from Charles River Laboratories. Female rats were superovulated with injection of 20 IU PMSG, followed by injection of 50 IU HCG and immediate mating to CD Sprague-Dawley studs $48 \mathrm{~h}$ later. The pronuclei of fertilized rat zygotes were injected with $40 \mathrm{ng} /$ $\mu \mathrm{L}$ Cas 9 mRNA and $20 \mathrm{ng} / \mu \mathrm{L}$ each of two sgRNAs. Surviving eggs were surgically transferred to CD Sprague-Dawley pseudopregnant females. All animal experiments were approved and performed in accordance with institutional IACUC protocols.

\section{Detecting the presence of indels by PCR-HMA and amplicon sequencing}

Genomic DNA from rat-tail biopsies was obtained by phenol extraction. DNA samples were directly used as a PCR template to amplify a region flanking the CRISPR target site. PCRs were set up using the following oligonucleotide primers: Ex20-F 5'-TCAACATGACTGGCTTCCTC-3' and Ex20R $5^{\prime}$-CATTGGATACAGAGCAGGACTC- $3^{\prime}$ to obtain a $284 \mathrm{bp}$ fragment. The amplicons were subjected to denaturation-slow renaturation to facilitate formation of heteroduplexes using a thermocycler. These samples were then resolved on polyacrylamide gels (10\%) and the resulting mobility profiles were used to infer efficiency of CRISPR-Cas9 nuclease activity. PCR using the same primers flanking CRISPR target site (Ex20-F and Ex20-R) was performed to amplify regions with indels (284 bp). The amplified products were cloned using the TOPO-TA cloning kit (Invitrogen, Carlsbad, CA). Ten representative colonies were picked from each plate and grown in $4 \mathrm{~mL}$ liquid cultures to isolate plasmid DNA. Plasmid DNA was sequenced using M13F and M13R primers.

\section{Genotyping}

Rat DNA was collected from tail clips by phenol extraction (Invitrogen 15593-031). Target regions of the DNA were PCR amplified using the forward primer rNF1-1A $5^{\prime}-$ CTTAGGCTGCAGAAAGTCTTC-3' and reverse primer rNF1-1B 5'-CTTCACCTGTCCTTGAGAGTC-3'. PCR products were 
digested with Hpy8I (Thermo Fisher Scientific). After digestion, DNA fragments were separated by electrophoresis on a $2 \%$ agarose gel and stained with ethidium bromide. Premature stop ( $439 \mathrm{bp}$ ) and in-frame (185 bp) mutations were identified by gel imaging.

\section{RT-PCR}

RNA was isolated from rat tissue by Trizol extraction (Invitrogen). RNA was treated with DNase I (Thermo Fisher Scientific \#18068015). Complementary DNA (cDNA) was prepared by using random primers (Life Technologies 48190-011) and SuperScript ${ }^{\text {TM }}$ II Reverse Transcriptase (Invitrogen 18064022). PCR was performed with forward primer (5'-TCAGTACACAACTTCTTGCC-3') and reverse primer (5'-GAACACGAACATATCTGACC- $3^{\prime}$ ) to create a $826 \mathrm{bp}$ amplicon. These PCR products were digested with the restriction enzyme Hpy8l as described above to identify mutations. Digested PS amplicons make a $745 \mathrm{bp}$ product and digested wild type make a $390 \mathrm{bp}$ and $368 \mathrm{bp}$ product. Digested PCR products were run on a 10\% TGX-PAGE gel (BioRad) and stained with ethidium bromide.

\section{Histology}

Tumors were fixed and processed following the previous methods ${ }^{59}$. DAKO PT Link was used for antigen retrieval. Immunohistochemical staining was done with ER (Thermo MA5-13304), HER2 (Thermo MA5-13105), Cytokeratin (DAKO AE1/AE3), PR (Thermo MA1-411), and pERalpha S118 (SAB4504399 Sigma-Aldrich) using the DAKO Autostainer Link 48 . Ki67 (Spring Bioscience SP6) was stained using the Ventana Discovery Ultra.

\section{Rat Embryo Fibroblast (REF) Isolation}

At embryonic day 13.5, embryos were harvested and isolated independently in petri dishes with PBS. The placenta was removed followed by the yolk sac. Embryos were then dissected by removing the head, organs, and blood vessels. The remaining tissue was transferred to a new petri dish and finely chopped using surgical blades. Tissue was then digested in $0.25 \%$ trypsin for $15 \mathrm{~min}$ at $37^{\circ} \mathrm{C}$. Cells were passed through a cell strainer to isolate single cells. Cells were plated and grown in DMEM (Gibco 11995065) supplemented with $10 \%$ FBS and $1 \%$ penicillin.

\section{Rat mammary epithelial cell (MEC) isolation}

Mammary glands from adult rats were dissected and minced. Single-cell suspensions were made by digestion of minced tissue with EpiCultB medium (StemCell Technologies), 5\% fetal bovine serum, containing 1:9 collagenase/hyaluronidase cocktail solution (StemCell Technologies) for $15 \mathrm{~h}$ at $37^{\circ} \mathrm{C}$. Samples were centrifuged and ammonium chloride (StemCell Technologies) in HBSS $+2 \%$ FBS (HF) was added to the pellet to lyse red blood cells. Organoids were isolated by differential centrifugation and were dissociated in $0.2 \%$ trypsin-EDTA for $5 \mathrm{~min}$ followed by $5 \mathrm{U} / \mathrm{ml}$ dispase (StemCell Technologies) with $0.1 \mathrm{mg} / \mathrm{ml}$ DNase I (StemCell Technologies) for $5 \mathrm{~min}$. Cells were filtered through a $40 \mu \mathrm{m}$ cell strainer. To separate epithelial cells from hematopoietic and endothelial cells, samples were subjected to Miltenyi Biotec's Dead Cell Removal kit followed by immunomagnetic cell selection using a Miltenyi quadroMACS magnet (Miltenyi Biotec). Antibodies for selection included CD31-biotin and CD45biotin (Miltenyi Biotec). The selection was carried out according to the manufacturer's protocol using an LS column and anti-biotin microbeads (Miltenyi Biotec) and selection was verified by flow cytometry analysis by comparing the pre $(\mathrm{CD} 45+, \mathrm{CD} 31+, \mathrm{CD} 24+, \mathrm{CD} 29+)$ and post-selected $(\mathrm{CD} 24+, \mathrm{CD} 29+)$ cell populations. To deplete stromal contaminants, eluted cells were plated at $2 \times 10^{6}$ cells $/$ plate on $100 \mathrm{~mm}$ plates for three days in DMEM/F12 (Gibco) containing: 10\% horse serum (Thermo Fisher Scientific), $20 \mathrm{ng} / \mathrm{mL}$ EGF (Sigma), $0.5 \mu \mathrm{g} / \mathrm{mL}$ hydrocortisone (Sigma), $100 \mathrm{ng} / \mathrm{mL}$ cholera toxin (Sigma), and $10 \mathrm{ug} / \mathrm{mL}$ insulin (Sigma). Minimal fibroblast contamination was verified by microscopically observing the cultures before harvesting

\section{Immunoblot analysis}

Rat tissue was homogenized with a hand held pellet pestle and rat mammary epithelial cells were lysed in RIPA buffer (20 mM TrisHCL, pH 7.6; $5 \mathrm{mM}$ EDTA; $150 \mathrm{mM} \mathrm{NaCl} ; 0.5 \%$ NP-40; $50 \mathrm{mM} \mathrm{NaF} ; 1 \mathrm{mM}$ betaglycerophosphate) supplemented with PhosSTOP (Roche) and protease inhibitors (Roche). Samples were resolved by SDS-polyacrylamide gel electrophoresis on a 7.5\% TGX-PAGE gel (BioRad) and transferred overnight at $4{ }^{\circ} \mathrm{C}$. Blots presented in figure panels were derived from the same experiment and were processed in parallel. Immunoblotting was performed using the following antibodies: neurofibromin H-12 (SC-376886) from Santa Cruz Technology; pERalpha (SAB4504399) from Sigma-Aldrich; ERalpha (MA5-13304) from Thermo; $\beta$-actin (\#3700), HSP90 (\#4877), pERK (\#4370), ERK (\#9102), pAKT (\#9271), AKT (9272), pSTAT3 (\#9145), STAT3 (\#9139), pMET (\#3077) and MET (\#3127) from Cell Signaling.

\section{Immunofluorescence}

For immunofluorescence, FFPE tumors and mammary fat pad sections were deparrafinized and underwent heat-induced epitope retrieval (EDTA/ borate/Tris) and stained using the Ventana Discovery Ultra (Ventana Medical Systems). Primary antibodies included pERK (\#4370), pS6 (\#2215), and PSTAT3 (\#9145) from Cell Signaling, along with PMET (ab5662) from Abcam. In addition to DAPI, primary antibodies were revealed with antirabbit Alexa-Fluor 594 (\#8889, Cell Signaling). Lack of non-specific staining of the secondary antibody was confirmed in a secondary-only control. Using a Nikon A1plus-RSi laser scanning confocal microscope, images were taken with a $10 \times$ objective. Confocal $z$-stacks were acquired with a $60 \times$ oil immersion objective. PMT levels were set using the control mammary fat pad. Three fields of view per sample were captured with Nikon software. Using FIJl, maximum projection intensity images were generated.

\section{Statistical analysis}

For analysis of Nf1 rat survival, data were plotted using Kaplan-Meier curves and $p$-values were calculated using a Cox mixed-effects model with a frailty term for litter (https://CRAN.R-project.org/package=coxme). Linear contrasts with Benjamini-Hochberg false discovery rate adjustments were used to test specific hypotheses. All analyses described below were performed using R v 3.4.0. (https://cran.r-project.org/) All hypotheses were two-sided with a significance level of 0.05 . Ki67 analysis was analyzed using a linear mixed-effects regression and a Wald test. Poisson regression, via the base $R$ function $\mathrm{glm}$, was used to determine if premature stop and in-frame deletion rats had significantly different tumor counts at euthanasia. Ovariectomy analysis was analyzed using a multi-level mixed-effects beta regression with a random intercept for tumor, clustered within rat lines. This model was fit using the $R$ package $\operatorname{glmmTMB}^{60}$ and the squeeze algorithm. ${ }^{61}$

\section{METABRIC analysis}

Survival analysis was conducted in $\mathrm{R} v(3.4 .2)$ using the packages survival (https://cran.r-project.org/web/packages/survival/index.html), survminer (https://cran.r-project.org/web/packages/survminer/index.html), and coxme. Multiple variables were assessed (Supplementary Table 4). Clinical, copy number, and gene expression data from the METABRIC data $\mathrm{set}^{33}$ were accessed using the cgdsr (https://cran.r-project.org/web/packages/ cgdsr/index.html) package in R. Patients were selected based on the availability of both copy number and gene expression data, and HER2 negative status. Geneco-expression network analysis was conducted using the WGCNA ( $\mathrm{v} 1.61$ ) package in R to identify gene co-expression modules from mRNA expression data (Illumina Human v3 Microarray). ${ }^{43}$ Data were normalized as previously described. Unsigned correlations were used with a soft threshold value $\beta$ of 10 and a treecut value of 0.15 , a minimum number of genes in the module set to 30 . The $\beta$ and treecut parameters were chosen after assessing the quality of modules detected, and all other parameters used default settings. Unsupervised clustering of WGCNA results was run using the pheatmap package in $R$ (v1.0.8). Logistic regression, via the base $R$ function $\mathrm{glm}$, was used to determine if identified clusters had significantly different rates of shallow deletion mutations. Specific gene networks were visualized using MetaCore from Thomson Reuters (v6.33 build 69110). The RAS and KRAS gene lists used in the analysis were provided by the Broad Institute MSigDB database (v6.1) Hallmark gene set collection and BioCarta (c) (2000-2017 BioCarta, all rights reserved). Module gene lists and processed METABRIC data are available at Synapse (ID: syn11946759).

\section{DATA AVAILABILITY}

The authors declare that the main data supporting the findings of this study are available within the article and its Supplementary Information files. Extra data are available from the corresponding author upon request. 


\section{ACKNOWLEDGEMENTS}

We dedicate this work in honor of Patricia Graveel and all neurofibromatosis patients dealing with breast cancer. We would like to thank Dr. Ben Johnson and Jamie Grit for their critical review of this manuscript, Cassie Diegel, Dr. Casey Droscha and Dr. Bart Williams for assistance with CRISPR designs, and also Lisa Turner (VAI Histology) and the VAI Vivarium staff for their strong work on this project. Funding for this research was made possible by the Breast Cancer Research Foundation (BCRF-17159), Muskegon Tempting Tables, and the VARI Faculty Innovation Award.

\section{AUTHOR CONTRIBUTIONS}

Study conception and design: P.S.D., C.R.G., M.R.S.; acquisition and analysis of data: P. S.D., E.A.T., C.J.E., E.J.E., Z.M., E.E.G., M.C., A.N.T, A.K.C., T.K., R.T.B., M.J.B., C.R.G., M.R.S.; provided reagents: B.E., R.A.K.; drafting of manuscript: C.R.G., M.R.S.; critical revision: P. S.D., E.A.T., Z.M., M.J.B.; C.R.G. and M.R.S. are the guarantors of this study.

\section{ADDITIONAL INFORMATION}

Supplementary information accompanies the paper on the npj Breast Cancer website (https://doi.org/10.1038/s41523-018-0080-8).

Competing interests: The authors declare no competing interests.

Publisher's note: Springer Nature remains neutral with regard to jurisdictional claims in published maps and institutional affiliations.

\section{REFERENCES}

1. Hanahan, D. \& Weinberg, R. A. Hallmarks of cancer: the next generation. Cell 144, 646-674 (2011)

2. Pylayeva-Gupta, Y., Grabocka, E. \& Bar-Sagi, D. RAS oncogenes: weaving a tumorigenic web. Nat. Rev. Cancer 11, 761-774 (2011).

3. Sivaraman, V. S., Wang, H., Nuovo, G. J. \& Malbon, C. C. Hyperexpression of mitogen-activated protein kinase in human breast cancer. J. Clin. Invest. 99 1478-1483 (1997).

4. von Lintig, F. C. et al. Ras activation in human breast cancer. Breast Cancer Res. Treat. 62, 51-62 (2000)

5. Mueller, $\mathrm{H}$. et al. Potential prognostic value of mitogen-activated protein kinase activity for disease-free survival of primary breast cancer patients. Int. J. Cancer 89, 384-388 (2000).

6. Bollag, G. et al. Loss of NF1 results in activation of the Ras signaling pathway and leads to aberrant growth in haematopoietic cells. Nat. Genet. 12, 144-148 (1996).

7. Upadhyaya, M. et al. Mutational and functional analysis of the neurofibromatosis type 1 (NF1) gene. Hum. Genet. 99, 88-92 (1997).

8. Ratner, N. \& Miller, S. J. A RASopathy gene commonly mutated in cancer: the neurofibromatosis type 1 tumour suppressor. Nat. Rev. Cancer 15, 290-301 (2015).

9. Lammert, M., Friedman, J. M., Kluwe, L. \& Mautner, V. F. Prevalence of neurofibromatosis 1 in German children at elementary school enrollment. Arch. Dermatol. 141, 71-74 (2005).

10. Evans, D. G. et al. Birth incidence and prevalence of tumor-prone syndromes: estimates from a UK family genetic register service. Am. J. Med. Genet. A. 152A, 327-332 (2010)

11. Seminog, O. O. \& Goldacre, M. J. Risk of benign tumours of nervous system, and of malignant neoplasms, in people with neurofibromatosis: population-based record-linkage study. Br. J. Cancer 108, 193-198 (2013).

12. Cancer Genome Atlas Research Network. Comprehensive genomic characterization defines human glioblastoma genes and core pathways. Nature 455, 1061-1068 (2008).

13. Cancer Genome Atlas Research Network. Integrated genomic analyses of ovarian carcinoma. Nature 474, 609-615 (2011).

14. Ding, L. et al. Somatic mutations affect key pathways in lung adenocarcinoma. Nature 455, 1069-1075 (2008).

15. Seminog, O. O. \& Goldacre, M. J. Age-specific risk of breast cancer in women with neurofibromatosis type 1. Br. J. Cancer 112, 1546-1548 (2015).

16. Uusitalo, E. et al. Distinctive cancer associations in patients with neurofibromatosis type 1. J. Clin. Oncol. 34, 1978-1986 (2016).

17. Howell, S. J., Hockenhull, K., Salih, Z. \& Evans, D. G. Increased risk of breast cancer in neurofibromatosis type 1: current insights. Breast Cancer (Dove Med. Press) 9, 531-536 (2017).

18. Stephens, P. J. et al. The landscape of cancer genes and mutational processes in breast cancer. Nature 486, 400-404 (2012).
19. Cancer Genome Atlas Network. Comprehensive molecular portraits of human breast tumours. Nature 490, 61-70 (2012).

20. Wallace, M. D. et al. Comparative oncogenomics implicates the neurofibromin 1 gene (NF1) as a breast cancer driver. Genetics 192, 385-396 (2012).

21. Bajenaru, M. L. et al. Astrocyte-specific inactivation of the neurofibromatosis 1 gene (NF1) is insufficient for astrocytoma formation. Mol. Cell. Biol. 22, 5100-5113 (2002).

22. Zhu, Y. et al. Inactivation of NF1 in CNS causes increased glial progenitor proliferation and optic glioma formation. Development 132, 5577-5588 (2005).

23. Cichowski, K. et al. Mouse models of tumor development in neurofibromatosis type 1. Science 286, 2172-2176 (1999).

24. Zhu, Y. et al. Early inactivation of p53 tumor suppressor gene cooperating with NF1 loss induces malignant astrocytoma. Cancer Cell. 8, 119-130 (2005).

25. Lin, J. H. Applications and limitations of genetically modified mouse models in drug discovery and development. Curr. Drug. Metab. 9, 419-438 (2008).

26. Brannan, C. I. et al. Targeted disruption of the neurofibromatosis type-1 gene leads to developmental abnormalities in heart and various neural crest-derived tissues. Genes Dev. 8, 1019-1029 (1994).

27. Silva, A. J. et al. A mouse model for the learning and memory deficits associated with neurofibromatosis type I. Nat. Genet. 15, 281-284 (1997).

28. Vogel, K. S. et al. Mouse tumor model for neurofibromatosis type 1. Science 286, 2176-2179 (1999).

29. Reilly, K. M., Loisel, D. A., Bronson, R. T., McLaughlin, M. E. \& Jacks, T. Nf1;Trp53 mutant mice develop glioblastoma with evidence of strain-specific effects. Nat. Genet. 26, 109-113 (2000).

30. Bajenaru, M. L. et al. Optic nerve glioma in mice requires astrocyte Nf1 gene inactivation and Nf1 brain heterozygosity. Cancer Res. 63, 8573-8577 (2003).

31. Russo, J. Significance of rat mammary tumors for human risk assessment. Toxicol. Pathol. 43, 145-170 (2015).

32. Aitman, T., Dhillon, P. \& Geurts, A. M. A RATional choice for translational research? Dis. Models 9, 1069-1072 (2016).

33. Curtis, C. et al. The genomic and transcriptomic architecture of 2,000 breast tumours reveals novel subgroups. Nature 486, 346-352 (2012).

34. Challa, A. K. et al. Novel hypomorphic alleles of the mouse tyrosinase gene induced by CRISPR-Cas9 nucleases cause non-albino pigmentation phenotypes. PLoS One 11, e0155812 (2016).

35. Lambert, L. J. et al. Increased trabecular bone and improved biomechanics in an osteocalcin-null rat model created by CRISPR/Cas9 technology. Dis. Model Mech. 9, 1169-1179 (2016).

36. Liu, R. Z., Li, X. \& Godbout, R. A novel fatty acid-binding protein (FABP) gene resulting from tandem gene duplication in mammals: transcription in rat retina and testis. Genomics 92, 436-445 (2008).

37. Aitman, T. J. et al. Copy number polymorphism in Fcgr3 predisposes to glomerulonephritis in rats and humans. Nature 439, 851-855 (2006).

38. Gutmann, D. H., Geist, R. T., Wright, D. E. \& Snider, W. D. Expression of the neurofibromatosis 1 (NF1) isoforms in developing and adult rat tissues. Cell Growth Differ. 6, 315-323 (1995).

39. Andersen, L. B. et al. A conserved alternative splice in the von Recklinghausen neurofibromatosis (NF1) gene produces two neurofibromin isoforms, both of which have GTPase-activating protein activity. Mol. Cell. Biol. 13, 487-495 (1993).

40. Gutmann, D. H., Geist, R. T., Rose, K. \& Wright, D. E. Expression of two new protein isoforms of the neurofibromatosis type 1 gene product, neurofibromin, in muscle tissues. Dev. Dyn. 202, 302-311 (1995).

41. Peacock, J. D. et al. Genomic status of MET potentiates sensitivity to MET and MEK inhibition in NF1-related malignant peripheral nerve sheath tumors. Cancer Res. 78, 3672-3687 (2018).

42. Torres, K. E. et al. Activated MET is a molecular prognosticator and potential therapeutic target for malignant peripheral nerve sheath tumors. Clin. Cancer Res. 17, 3943-3955 (2011).

43. Langfelder, P. \& Horvath, S. WGCNA: an R package for weighted correlation network analysis. BMC Bioinforma. 9, 559 (2008).

44. Hurtado, A., Holmes, K. A., Ross-Innes, C. S., Schmidt, D. \& Carroll, J. S. FOXA1 is a key determinant of estrogen receptor function and endocrine response. Nat. Genet. 43, 27 (2010).

45. Badve, S. et al. FOXA1 expression in breast cancer-correlation with luminal subtype A and survival. Clin. Cancer Res. 13, 4415-4421 (2007).

46. Thakkar, A. et al. High expression of three-gene signature improves prediction of relapse-free survival in estrogen receptor-positive and node-positive breast tumors. Biomark. Insights 10, 103-112 (2015). BMI.S30559.

47. Lopez-Bergami, P., Lau, E. \& Ronai, Z. E. Emerging roles of ATF2 and the dynamic AP1 network in cancer. Nat. Rev. Cancer 10, 65 (2010).

48. Collins, L. C. et al. Androgen receptor expression in breast cancer in relation to molecular phenotype: results from the Nurses' Health Study. Mod. Pathol. 24, 924-931 (2011). 
49. Ciupek, A. et al. Androgen receptor promotes tamoxifen agonist activity by activation of EGFR in ERalpha-positive breast cancer. Breast Cancer Res. Treat. 154, 225-237 (2015)

50. D'Amato, N. C. et al. Cooperative dynamics of AR and ER activity in breast cancer. Mol. Cancer Res. 14, 1054-1067 (2016).

51. Kato, S. et al. Activation of the estrogen receptor through phosphorylation by mitogen-activated protein kinase. Science 270, 1491-1494 (1995).

52. Le Romancer, M. et al. Cracking the estrogen receptor's posttranslational code in breast tumors. Endocr. Rev. 32, 597-622 (2011).

53. McLaughlin, S. K. et al. The RasGAP gene, RASAL2, is a tumor and metastasis suppressor. Cancer Cell. 24, 365-378 (2013).

54. Chang, E. et al. Abstract GS2-02: direct regulation of estrogen receptor-a (ER) transcriptional activity by NF1. Cancer Res. 78, GS2-02 (2018).

55. Yates, L. R. et al. Genomic evolution of breast cancer metastasis and relapse. Cancer Cell. 32, 169-184 e7 (2017)

56. Stricker, T. P. et al. Robust stratification of breast cancer subtypes using differential patterns of transcript isoform expression. PLoS. Genet. 13, e1006589 (2017).

57. Thomson, S. A. \& Wallace, M. R. RT-PCR splicing analysis of the NF1 open reading frame. Hum. Genet. 110, 495-502 (2002).

58. Vandenbroucke, I., Vandesompele, J., De Paepe, A. \& Messiaen, L. Quantification of NF1 transcripts reveals novel highly expressed splice variants. FEBS Lett. 522, 71-76 (2002).
59. Linklater, E. S. et al. Targeting MET and EGFR crosstalk signaling in triple-negative breast cancers. Oncotarget 7, 69903-69915 (2016).

60. Brooks, M. E. et al. Modeling zero-inflated count data with glmmTMB. bioRxiv (2017) https://doi.org/10.1101/132753.

61. Smithson, M. \& Verkuilen, J. A better lemon squeezer? Maximum-likelihood regression with beta-distributed dependent variables. Psychol. Methods 11, 54-71 (2006).

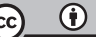

Open Access This article is licensed under a Creative Commons Attribution 4.0 International License, which permits use, sharing, adaptation, distribution and reproduction in any medium or format, as long as you give appropriate credit to the original author(s) and the source, provide a link to the Creative Commons license, and indicate if changes were made. The images or other third party material in this article are included in the article's Creative Commons license, unless indicated otherwise in a credit line to the material. If material is not included in the article's Creative Commons license and your intended use is not permitted by statutory regulation or exceeds the permitted use, you will need to obtain permission directly from the copyright holder. To view a copy of this license, visit http://creativecommons. org/licenses/by/4.0/.

(c) The Author(s) 2018 